\title{
YAP-dependent necrosis occurs in early stages of Alzheimer's disease and regulates mouse model pathology
}

\author{
Hikari Tanaka1,14, Hidenori Homma,14, Kyota Fujita1,14, Kanoh Kondo1,14, Shingo Yamada 2,14, Xiaocen Jin', \\ Masaaki Waragai (i) ${ }^{3}$, Gaku Ohtomo ${ }^{4}$, Atsushi Iwata4, Kazuhiko Tagawa', Naoki Atsuta ${ }^{5}$, Masahisa Katsuno (1) ${ }^{5}$, \\ Naoki Tomita ${ }^{6}$, Katsutoshi Furukawa ${ }^{6}$, Yuko Saito ${ }^{7}$, Takashi Saito ${ }^{8}$, Ayaka Ichise ${ }^{9}$, Shinsuke Shibata (D) ${ }^{9}$, \\ Hiroyuki Arai ${ }^{6}$, Takaomi Saido ${ }^{8}$, Marius Sudol ${ }^{10}$, Shin-ichi Muramatsu (1D ${ }^{11}$, Hideyuki Okano ${ }^{9}$, Elliott J. Mufson ${ }^{12}$, \\ Gen Sobue (1) ${ }^{5}$, Shigeo Murayama ${ }^{13} \&$ Hitoshi Okazawa ${ }^{1 \star}$
}

The timing and characteristics of neuronal death in Alzheimer's disease (AD) remain largely unknown. Here we examine $A D$ mouse models with an original marker, myristoylated alanine-rich C-kinase substrate phosphorylated at serine 46 (pSer46-MARCKS), and reveal an increase of neuronal necrosis during pre-symptomatic phase and a subsequent decrease during symptomatic phase. Postmortem brains of mild cognitive impairment $(\mathrm{MCl})$ rather than symptomatic $A D$ patients reveal a remarkable increase of necrosis. In vivo imaging reveals instability of endoplasmic reticulum (ER) in mouse $A D$ models and genome-edited human AD iPS cell-derived neurons. The level of nuclear Yes-associated protein (YAP) is remarkably decreased in such neurons under $A D$ pathology due to the sequestration into cytoplasmic amyloid beta $(A \beta)$ aggregates, supporting the feature of YAP-dependent necrosis. Suppression of early-stage neuronal death by AAV-YAPdeltaC reduces the laterstage extracellular $A \beta$ burden and cognitive impairment, suggesting that preclinical/prodromal YAP-dependent neuronal necrosis represents a target for AD therapeutics.

\footnotetext{
${ }_{1}^{1}$ Department of Neuropathology, Medical Research Institute and Center for Brain Integration Research, Tokyo Medical and Dental University, 1-5-45 Yushima, Bunkyo-ku, Tokyo 113-8510, Japan. ${ }^{2}$ Shino-Test Corporation, 2-29-14, Ohino-dai, Minami-ku, Sagamihara, Kanagawa 252-0331, Japan.

${ }^{3}$ Department of Neurology, Higashi Matsudo Municipal Hospital, Matsudo, Chiba 270-2222, Japan. ${ }^{4}$ Department of Neurology, The University of Tokyo, Graduate School of Medicine, 7-3-1 Hongo, Bunkyo-ku, Tokyo 113-8655, Japan. ${ }^{5}$ Department of Neurology, Brain and Mind Research Center, Nagoya University Graduate School of Medicine, 65 Tsurumai-cho, Showa-ku, Nagoya Aichi 466-8550, Japan. 6 Department of Geriatrics \& Gerontology, Division of Brain Science, Institute of Development, Aging and Cancer, Tohoku University, 4-1, Seiryo-cho, Aoba-ku, Sendai 980-8575, Japan. ${ }^{7}$ Department of Laboratory Medicine, National Center Hospital, National Center of Neurology and Psychiatry, 4-1-1 Ogawa-Higahsi-machi, Kodaira, Tokyo, Japan.

${ }^{8}$ Laboratory for Proteolytic Neuroscience, RIKEN Center for Brain Science, 2-1 Hirosawa, Wako, Saitama 351-0198, Japan. ${ }^{9}$ Department of Physiology, Keio University School of Medicine, 35 Shinano-machi, Shinjuku-ku, Tokyo 160-8582, Japan. ${ }^{10}$ Department of Physiology, National University of Singapore, Yong Loo Li School of Medicine, 2 Medical Drive, Singapore 117597, Singapore. ${ }^{11}$ Department of Neurology, Jichi Medical University, 3311-1 Yakushiji, Shimotsuke, Tochigi 329-0496, Japan. ${ }^{12}$ Department of Neurobiology and Neurology, Barrow Neurological Institute, 350 W. Thomas Road, Phoenix, AZ 85013 , USA ${ }^{13}$ Department of Neuropathology, Brain Bank for Aging Research, Tokyo Metropolitan Institute of Gerontology, 35-2, Sakae-cho, Itabashi-ku, Tokyo 173-0015, Japan. ${ }^{14}$ These authors contributed equally: Hikari Tanaka, Hidenori Homma, Kyota Fujita, Kanoh Kondo, Shingo Yamada. *email: okazawa.npat@mri.tmd.ac.jp
} 
T he ability to diagnose $\mathrm{AD}$ at an early stage is eagerly anticipated, especially after clinical trials of anti-A $\beta$ antibodies $^{1,2}$ and $\gamma$-/ $/ \beta$-secretase inhibitors ${ }^{3,4}$ in post-onset patients proved disappointing. A deeper understanding of MCI could play a pivotal role in the development of new therapeutic strategies for AD. Despite the importance of MCI, the pathological and molecular evaluation remains insufficient especially from the aspect of chronological change of neuronal function and cell death. Accordingly, no efficient single biomarker directly reflecting disease activity in $\mathrm{MCI}$ has yet been reported.

Cutting-edge techniques, including comprehensive analyses, have identified molecules in addition to $A \beta$ and tau that could be targeted for therapeutic intervention at the early stage of $\mathrm{AD}$. For instance, comparison of neuroimaging and transcriptome data revealed that a genetic profile of lipid metabolism centered by APOE affects propagation patterns of both $A \beta$ and tau in the brain $^{5}$. In another study, a meta-analysis of functional genomic data from $\mathrm{AD}$ showed that YAP, a co-transcriptional factor that regulates cell death and survival by binding to the different transcription factors p73 and TEA domain family member 1 (TEAD) ${ }^{6-9}$, is positioned at the center of the molecular network of $\mathrm{AD}^{10}$. Elevated activity of TEAD mediated by YAP has been implicated in cell proliferation, differentiation, and survival ${ }^{11-13}$, whereas elevated p73 $14-16$ activity and reduced TEAD ${ }^{17-19}$ activity promote apoptosis and necrosis, respectively.

Previously, we performed a comprehensive phosphoproteome analysis of four strains of $\mathrm{AD}$ model mice and human postmortem $\mathrm{AD}$ brains, and discovered three proteins whose phosphorylation state is altered at a very early stage before extracellular amyloid aggregate ${ }^{20}$. One such protein is MARCKS, which anchors the actin cytoskeleton to the plasma membrane and plays a critical role in stabilizing the post-synaptic structure of dendritic spines ${ }^{21}$. Phosphorylation of MARCKS at Ser46 decreases its affinity for actin and destabilizes dendritic spines ${ }^{22}$. High mobility group box-1 (HMGB1) contributes to the MARCKS phosphorylation via Toll-like receptor 4 (TLR4) since blockade of HMGB1-TLR4 binding with monoclonal anti-HMGB1 antibodies suppresses the phosphorylation of MARCKS at Ser46, stabilizes dendritic spines, and rescues cognitive impairment in $\mathrm{AD}$ model mice ${ }^{22}$. Given that HMGB1 is released from necrotic cells ${ }^{23,24}$, it remains unclear how MARCKS phosphorylation, which occurs at the early stage of $\mathrm{AD}$ pathology, is connected to neuronal cell death, which is believed to occur at a relatively late stage.

In this study, we found that HMGB1 levels were remarkably elevated in CSF of MCI, but not so elevated in AD patients. Consistent with this, active neuronal necrosis revealed by pSer46MARCKS increased to the greatest extent during preclinical stages of $\mathrm{AD}$ mouse models and human MCI patients. In addition, we showed that the observed necrosis was caused by a deficiency of YAP, resulting in suppression of the transcriptional activity of TEAD, the final effector molecule of the Hippo pathway ${ }^{11-13}$, in mouse AD models, human AD iPS neuron models and human postmortem MCI brains. These findings unravel the occurrence of cell death at the early stage in $\mathrm{AD}$, which could be a therapeutic target that prevents progression of $\mathrm{AD}$.

\section{Results}

HMGB1 is elevated in CSF of human MCI patients. CSF samples were collected by lumbar puncture from 34 normal controls, 14 disease controls, $26 \mathrm{MCI}$ patients, and $73 \mathrm{AD}$ patients (Supplementary Tables 1,2 ). MCI and AD were diagnosed by ICD-10, and the patients were categorized as having amnestic MCI. There was no significant difference in age between the different patient groups, but the proportion of female patients was slightly higher in the AD group than in the other groups (Supplementary
Table 1). ApoE subtyping was performed in $19 \mathrm{MCI}$ and $18 \mathrm{AD}$ patients (Supplementary Table 1). In the disease control patients, CSF samples were taken because neurological diseases were suspected; therefore, there was some bias in the types of disease present in this patient group (Supplementary Table 2). To verify the accuracy of MCI/AD diagnosis, we compared the levels of $\mathrm{A} \beta 42, \mathrm{pTau}$, and $\mathrm{pTau} / \mathrm{A} \beta 42$ between the normal control group and the $\mathrm{MCI}$ or $\mathrm{AD}$ group. In support of the clinical diagnoses, $A \beta 42$ levels were reduced, and $p T a u / A \beta 42$ levels were elevated, in the MCI and AD groups (Supplementary Fig. 1). APP/A $\beta$ ratio was increased in $\mathrm{AD}$ group in comparison to other groups (Supplementary Fig. 1), as reported previously ${ }^{25}$.

Expecting elevation of HMGB1 in symptomatic $\mathrm{AD}$, we evaluated HMGB1 concentrations in CSF by ELISA. However, the CSF-HMGB1 level was significantly elevated in the clinically diagnosed $\mathrm{MCI}$ group, but not the $\mathrm{AD}$ group, relative to the normal or disease controls (Fig. 1a). The CSF-HMGB1 level was also significantly higher in the MCI group than in the $\mathrm{AD}$ group (Fig. 1a). In receiver operating characteristic (ROC) analysis of the comparisons between MCI and the normal or disease controls, the area under the curve (AUC) was 0.861 or 0.931 , respectively (Fig. 1b). In addition, AUC was 0.809 in comparison between MCI and $\mathrm{AD}$, suggesting the CSF-HMGB1 value may assist clinical diagnosis of the two phenotypic states (Fig. 1b). Interestingly, we observed no significant correlations between CSF-HMGB1 and Mini-Mental State Examination (MMSE) score in the MCI, AD, or MCI + AD group (Supplementary Fig. 2).

In the MCI group, we observed a positive relationship between levels of CSF-HMGB1 and levels of $A \beta 42, A \beta 40$, and tau (Supplementary Fig. 3). Levels of pTau were not related to levels of CSF-HMGB1 in MCI patients (Supplementary Fig. 3). Moreover, we detected no relationship between CSF-HMGB1 and $\mathrm{A} \beta 42, \mathrm{~A} \beta 40$, tau, or pTau in the AD group (Supplementary Fig. 3). The number of patients in which both $A \beta 42$ and $A \beta 40$ could be analyzed was small, so this result is not informative (Supplementary Fig. 3).

We observed no relationship between ApoE4 allele copy number and CSF-HMGB1 in the MCI group. However, ApoE4 was negatively correlated with CSF-HMGB1 in the AD group (Supplementary Fig. 3). This finding may be of interest, assuming that the summative pathology linked to CSF-HMGB1 and ApoE4 allele copy number reflects cognitive impairment.

Necrosis occurs most actively in the MCI stage. HMGB1 is a representative damage-associated molecular patterns (DAMPs) molecule released from necrotic cells ${ }^{23,24}$. Our findings in human CSF suggested that neuronal necrosis might occur more frequently in preclinical MCI than in symptomatic AD. Evaluation of cell death in vivo has been technically difficult because intensities of cell death markers diminish rapidly after cell death or are cleared by phagocytes in the brain. To overcome the difficulty, we employed anti-pSer46-MARCKS antibody whose reactivity was characterized by western blot ${ }^{22}$. The specificity to pSer46-MARCKS was further confirmed by ELISA using phospho- and non-phospho peptides matching to the 14 amino acid sequence of MARCKS around Ser46 (Supplementary Fig. 4a). Anti-pSer46-MARCKS antibody was purified with affinity columns of non-phosphorylated antigen peptide and the phosphorylated antigen peptide (Supplementary Fig. 4b). Therefore, we compared the reactivity of anti-pSer46-MARCKS antibody and anti-non-phosphorylated MARCKS antibody in immunohistochemistry of cerebral cortex from $5 x F A D$ mice at 6 months (Supplementary Fig. 4c). Obviously the patterns were different, and anti-pSer46-MARCKS antibody, but not antinon-phosphorylated MARCKS antibody, stained structures 
a

$$
\begin{aligned}
& p \text {-value: Wilcoxon's rank sum test with post-hoc } \\
& \text { Bonferroni correction }
\end{aligned}
$$

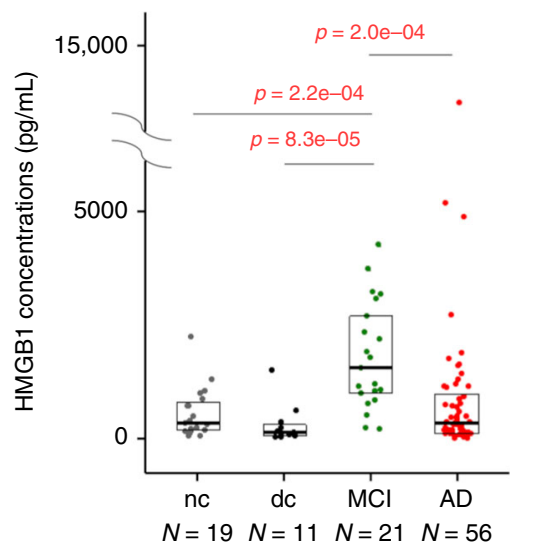

$\mathrm{nc}=$ normal control, $\mathrm{dc}=$ disease control. b
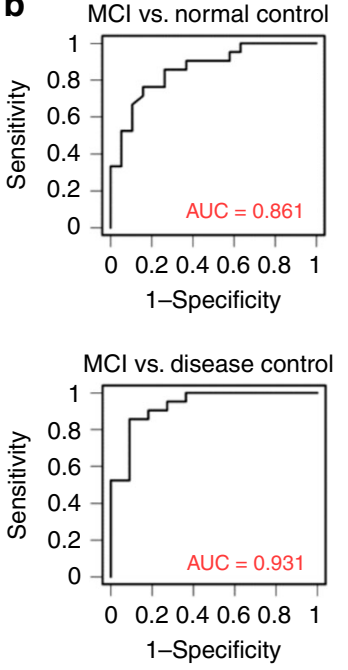
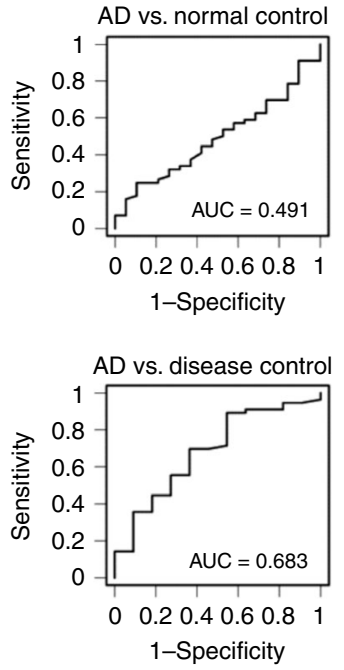

$\mathrm{AD}$ vs. $\mathrm{MCl}$

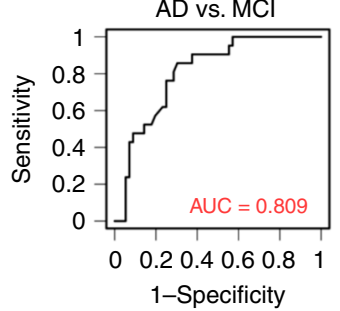

Fig. 1 HMGB1 levels are elevated in the CSF of $\mathbf{M C l}$ and AD patients. a CSF-HMGB1 levels in the normal control ( $\mathrm{nc}$ ) ( $N=19$ persons), disease control (dc) ( $N=11$ persons), $\mathrm{MCl}(N=21$ persons), and $A D(N=56$ persons) groups were evaluated by high-sensitivity ELISA. The box plot shows the median and quartiles. Statistical differences among groups were evaluated using the Wilcoxon rank-sum test with post-hoc Bonferroni correction. $\mathbf{b}$ Receiver operating characteristic (ROC) curves for the $\mathrm{MCl}$ or $\mathrm{AD}$ group versus the normal control (nc) and disease control (dc) groups. Area under the ROC curve $(A \cup C)$ values are shown in the graphs. Source data are provided as a "Source Data file".

around extracellular A $\beta$ aggregates (Supplementary Fig. 4c). We also performed western blot to examine chronological change of pSer46-MARCKS in the cerebral cortex of 5xFAD mice from 1 to 12 months (Supplementary Fig. 4d). Interestingly, pSer46MARCKS formed high molecular weight smear (HMW), suggesting that the character of MARCKS as an intrinsically disordered/denatured protein (IDP) 22,26 (Supplementary Fig. 4e) was enhanced by phosphorylation at pSer46. HMW smear, $80 \mathrm{kD}$ and $50 \mathrm{kD}$ bands were all increased during pathological progression (Supplementary Fig. 4d, right graph). In addition, pSer46-MARCKS was increased also during normal aging of non-transgenic sibling mice (Supplementary Fig. 4d, right graph). Consequently, the ratio of pSer46-MARCKS between $5 x F A D$ mice and non-transgenic sibling mice were declined after 3 months, consistently with our previous result of the similar ratio in mass analysis ${ }^{22}$.

pSer46-MARCKS reactivity increased in neurons surrounding dying cells, enabling us to detect active neuronal necrosis at the moment of dissection ${ }^{22}$ (Fig. 2a). Neurons under such active necrosis were marked by deformed and/or shrinking nuclei, sometimes with faint DAPI staining surrounded by degenerative neurites reactive for pSer46-MARCKS (Fig. 2a). Consistent with this, immunoelectron microscopy (IEM) confirmed that degenerative neurites reactive to pSer46-MARCKS antibody and full of autophagosomes surrounded intracellular $A \beta$ plaques (Supplementary Fig. 5a, b). Beyond the borders of degenerative neurites (Supplementary Fig. 5a, yellow dot lines), amyloid plaques included cytoplasmic organelles (Supplementary Fig. 5a, white arrows). Immunohistochemistry also revealed that the similar degenerative neurites surrounded non-apoptotic dying neurons (no chromatin condensation) with a deforming and shrinking nucleus (Supplementary Fig. 5c). These findings indicated that neurons died by necrosis at the center of degenerative neurite clusters, $A \beta$ persisting after cell death served as a seed for further extracellular amyloid aggregation, and that such necrotic neurons released DAMPs such as HMGB1, tau, and A $\beta$. Moreover, by using primary mouse cortical neurons, we confirmed that neurons under $\alpha$-amanitin-induced necrosis ${ }^{17}$ but not glutamateinduced apoptosis ${ }^{27-29}$ induced reactive increase of pSer46MARCKS in surviving neurons in neighborhood (Supplementary Fig. 6a). Western blot also supported induction of pSer46MARCKS in neurons by $a$-amanitin-induced necrosis but not glutamate-induced apoptosis (Supplementary Fig. 6b). These findings further supported that reactive pSer46-MARCKS in neighboring cells could be used as a marker specifically indicating necrotic change of the central neuron which they surrounded.

In this work, we strictly defined "active necrosis" as a single dying cell surrounded by reactive pSer46-MARCKS signals (Fig. 2a). Since necrotic cells or apoptotic cells not removed by phagocytes are known to trigger secondary necrosis ${ }^{30-32}$, we defined "secondary necrosis" as a cluster of multiple cells with reactive pSer46-MARCKS signals (Fig. 2a). Most extracellular A $\beta$ aggregates in $5 \mathrm{xFAD}$ mice were associated with pSer46-MARCKS and DAPI signals. However, in aged mice, a small part of A $\beta$ aggregates show disappearance or weakening of pSer46-MARCKS and DAPI signals, which we named as "ghost of cell death".

We found that the proportion of active necrosis increased during the preclinical stage of $5 x F A D$ mice ${ }^{33}$, from 1 to 6 months, and then decreased from 12 to 18 months after the onset of cognitive impairment (Fig. 2b). A similar relationship between 
a

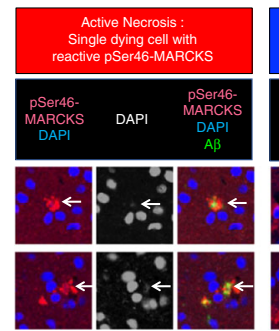

b
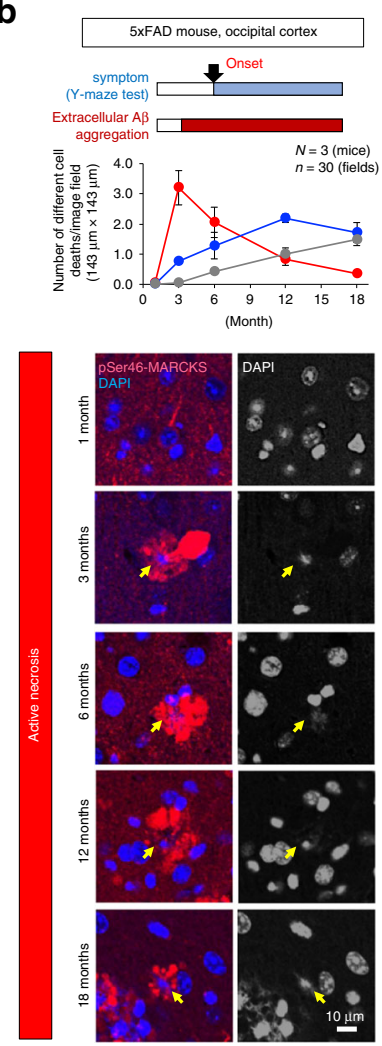

d

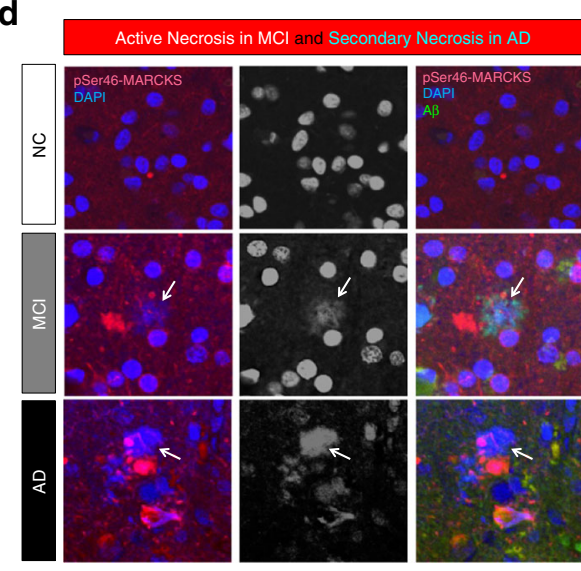

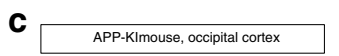
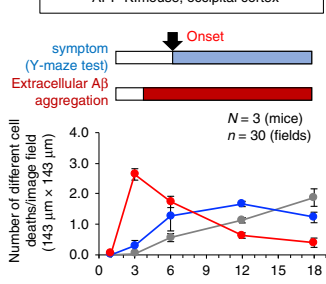

(Month)

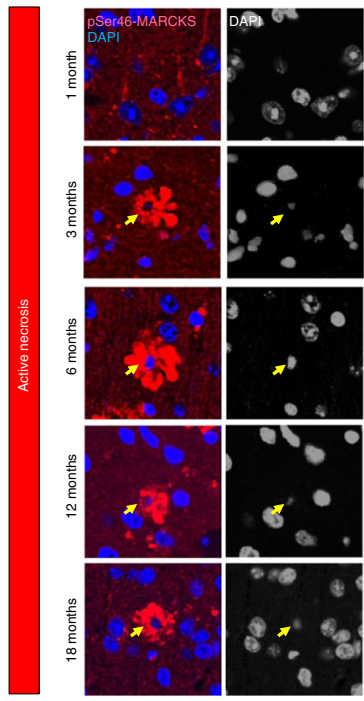

e

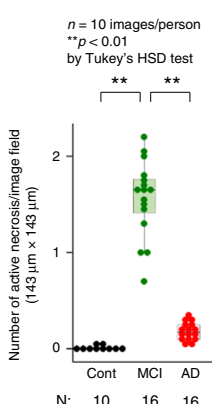

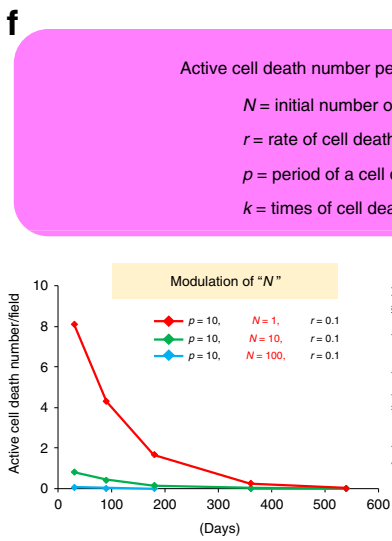
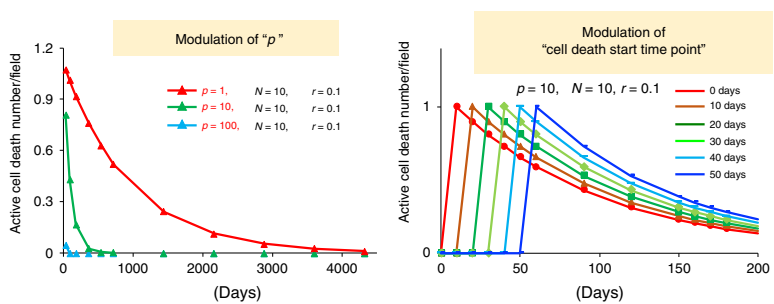

9

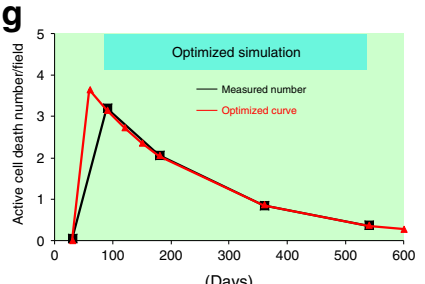

h
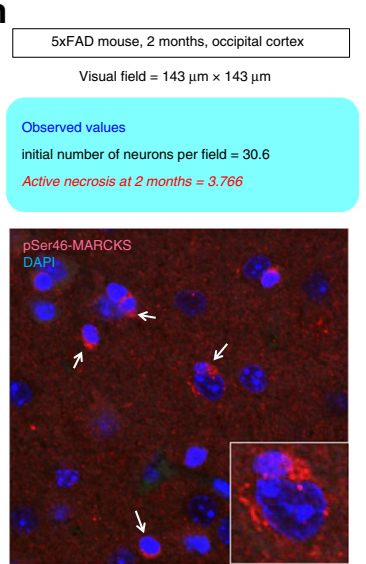

clinical stage and active necrosis was confirmed in human mutant APP knock-in mice (APP-KI mice) ${ }^{34}$ (Fig. 2c). This finding that cell death precedes extracellular $A \beta$ aggregate appearance at 3 months in $5 x F A D$ mice ${ }^{33}$ and at 4 months in APP-KI mice ${ }^{34}$ was unexpected. However, the early-stage appearance of active necrosis in mouse models (Fig. 2b, c) explains the elevation in the CSF-HMGB1 level in human MCI (Fig. 1) and presumably agrees with results of previous clinical trials.
Immunostaining of pSer46-MARCKS with postmortem human brains of MCI and non-neurological disease control patients confirmed that cortical neurons underwent morphologically similar necrosis in MCI patient brains (Fig. 2d). The active necrosis revealed by pSer46-MARCKS were present at significantly higher frequencies in all MCI patients than in AD patients (Fig. 2d, e), and neurons surrounded with reactive pSer46MARCKS, even though they did not match the strict criteria of 
Fig. 2 Necrosis occurs most actively at the preclinical stage in mouse and human AD brains. a Morphological definition of active necrosis, secondary necrosis and ghost of cell death. Active necrosis is specified by a single degraded nucleus detected by DAPI surrounded by pSer46-MARCKS-positive degenerative neurites. Secondary necrosis is a cluster of multiple dying cells with residual A $\beta$ in extracellular space and surrounding pSer46-MARCKS stains. Ghost of cell death is an extension of secondary necrosis in which DAPI and pSer46-MARCKS stains have faded out. b Upper graphs show time course of active necrosis, secondary necrosis and ghost of cell death in 5xFAD mice, in retrosplenial dysgranular cortex. Representative images from each time point are shown below the graph. Yellow arrow indicates a single degraded nucleus surrounded by reactive pSer46-MARCKS stains. $N=3$ mice, $n=$ 30 fields. c Time course of active necrosis in human mutant APP knock-in mice. Time course of necrosis $(N=3$ mice, $n=30$ fields) and representative images are shown. d Representative images of active necrosis in human $\mathrm{MCl}(\mathrm{MCl})$ and non-neurological disease control (NC). Rupturing or deformed nucleus undergoing necrosis is surrounded by $A \beta$ and pSer46-MARCKS-positive degenerative neurites (white arrow). e The box plot shows the number of active necrosis per visual field in the median, quartiles and whiskers that represent $1.5 \times$ the interquartile range. ${ }^{\star \star} p<0.01$, Tukey's HSD test $(n=10$ images/person). $\mathbf{f}$ Simulation of active necrosis. A formula was generated by assuming that cell death occurs at a constant rate in the residual neurons and in regular time interval (top). Modulation of each parameter changed simulation curves (graphs). $\mathbf{g}$ Numerical simulation program generated the optimized curve (red line) based on observed values of active necrosis in occipital cortex of 5xFAD mice (black line) and predicted parameter values and active necrosis at an unmeasured time point ( 2 months). $\mathbf{h}$ The number of active necrosis observed afterwards with samples at 2 months (60 days) matched exactly with the predicted number. Values in each group are summarized by mean \pm S.E.M. Source data are provided as a "Source Data file".

active necrosis because the nuclear DAPI stain remained intact, were increased in MCI (Supplementary Fig. 7). The number of neurons by itself was decreased in $\mathrm{AD}$ in comparison to that in MCI (Supplementary Fig. 7), and neurons surrounded with reactive pSer46-MARCKS were remarkably decreased in symptomatic AD.

Mathematical simulation of active necrosis. The chronological change of active necrosis motivated us to mathematically simulate chronological changes of active necrosis (Fig. 2f). If total number of neurons at the initial time point is $N$, and cell death (active necrosis) occurs constantly at the rate of $r$, residual number of neurons at current time $\left(N_{k}\right)$ is calculated as follows.

$$
N_{k}=N(1-r)^{k-1}
$$

Here, $k$ is the number of cell death cycles, and $k$ is calculated by the period necessary for a single turn of cell death and the time from the initial time point when cell death starts to the current time point.

Then, active cell death is calculated as following.

$$
\text { Active cell death }=N_{k}-N_{k-1}=N r(1-r)^{k-1}
$$

The simulation curve changed when the parameters, $N, r, p$ and the initial detection time point ( $p$ days later than initiation time point of cell death) were modulated (Fig. 2f).

As the graph shows, chronological change of actually observed active necrosis was precisely simulated (Fig. 2g). The consistency between theoretical and experimental data was surprising. The parameter deduced from observed number of active necrosis suggested that cell death period is 31 days and cell death ratio is 0.141 ( $14.1 \%$ of cell death die in 31 days). Initial number of neurons (30.3 cells) matched exactly with the neuronal number actually observed (30.6 cells) (Fig. $2 \mathrm{~g}$ ).

In addition, the mathematical simulation predicted that active necrosis process initiates from 1 month when intracellular $A \beta$ begins to be detected in immunohistochemistry ${ }^{22}$ and it should reach to 3.706 cells per area $(143 \mu \mathrm{m} \times 143 \mu \mathrm{m})$ at 2 months (Fig. 2g). Therefore, we examined again the brains of $5 x F A D$ mice at 2 months, and surprisingly found that the actual frequency active necrosis (3.766 cells/area) matched exactly with the expected value (Fig. 2h). These consistencies in the mathematical induction and deduction further supported our theory for dynamics of active necrosis.

ER enlargement is a morphological feature of necrosis in MCI. To characterize necrosis in vivo, we employed two-photon microscopy ${ }^{19}$ and analyzed dynamic changes of the ER in cortical neurons of 5xFAD mice from 1 (pre-symptomatic/preclinical stage) to 6 months (symptomatic/clinical stage) (Fig. 3a, b). The ER and A $\beta$ were visualized using ER-Tracker ${ }^{\mathrm{Tm}}$ and BTA1, respectively. At 1 month, ER volume was larger and less stable in $5 \mathrm{xFAD}$ than in non-transgenic sibling mice (B6/SJL) (Fig. 3a, b), and this tendency persisted at later time points (Fig. 3b, Supplementary Fig. 8). Moreover, these mice had a higher standard deviation or quartile deviation of ER volume, indicating that the ER was unstable in $5 \mathrm{xFAD}$ mice from 1 to 6 months (Fig. 3c). After two-photon microscopy, the mouse brains were investigated by electron microscopy. ER enlargement was confirmed at high frequencies in neurons of $5 \mathrm{xFAD}$ mice but rarely in nontransgenic sibling mice (B6/SJL) (Fig. 4a).

We extended electron microscopic analysis to human brains of non-neurological disease, MCI and AD patients (Fig. 4b). Remarkable enlargement of ER equivalent to the finding in $5 \times$ FAD mice was detected in MCI at a higher frequency than in $\mathrm{AD}$ patients (Fig. 4b). Instead, the frequency of extracellular aggregates was increased in $\mathrm{AD}$ (Fig. $4 \mathrm{~b}$, asterisk). Larger magnification revealed ribosomes on ER membrane confirming the origin of the ballooned organelles (Fig. 4c). A few ribosomes remained on the surface of extremely enlarged vacuoles (Fig. 4b, arrows in \#1 and \#2 of MCI) indicating that they originated from rough ER. Consistently, immunohistochemistry with anti-MAP2 and anti-calnexin (ER membrane marker) antibodies or with anti-MAP2 and anti-KDEL (ER content marker) antibodies revealed ER enlargement in cortical neurons of MCI patients (Fig. 4d) and of pre-symptomatic 5xFAD mice (Fig. 4e).

Intracellular $\mathbf{A} \boldsymbol{\beta}$ deprives YAP from the nucleus. The ER enlargement and instability we observed in 5xFAD mice were reminiscent of transcriptional repression-induced atypical cell death (TRIAD), the Hippo pathway-dependent necrosis ${ }^{17-19}$. Hence, we investigated key molecules in the Hippo pathway in human postmortem brains of MCI (amnestic MCI with AD pathology) and symptomatic AD patients. First, we discovered that intracellular $A \beta$ aggregates deprived YAP from the nucleus, ultimately causing a decrease in nuclear YAP levels in the cortical neurons of $\mathrm{AD}$ and MCI patients (Fig. 5a). This remarkable finding was observed in three MCI (amnestic MCI with AD pathology) and three symptomatic $\mathrm{AD}$ patients (Fig. 5b, upper graph). In MCI, DAPI signal intensities were decreased in neurons where intracellular $\mathrm{A} \beta$ aggregates deprived YAP from the nucleus (Fig. 5b, middle graph). Comparison among control, $\mathrm{MCI}$ and $\mathrm{AD}$ also confirmed decrease of DAPI signal intensities in cortical neurons with cytoplasmic YAP/A $\beta$-colocalization (Fig. 5b, lower graph). Immunoprecipitation of cerebral cortex tissues (temporal lobe) from human AD patients who had been 
a
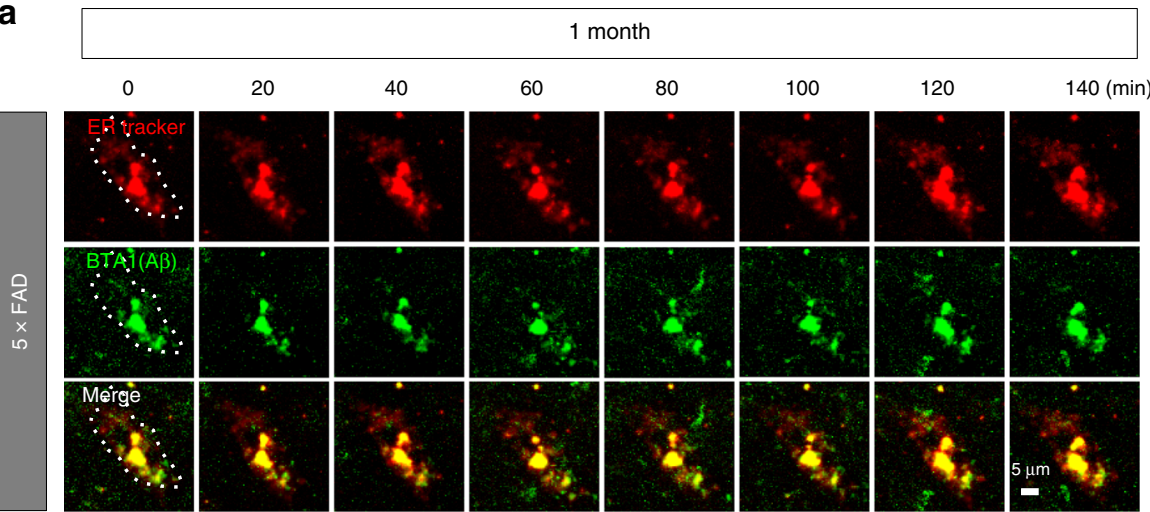

$60 \quad 80$

100

120

140 (min)
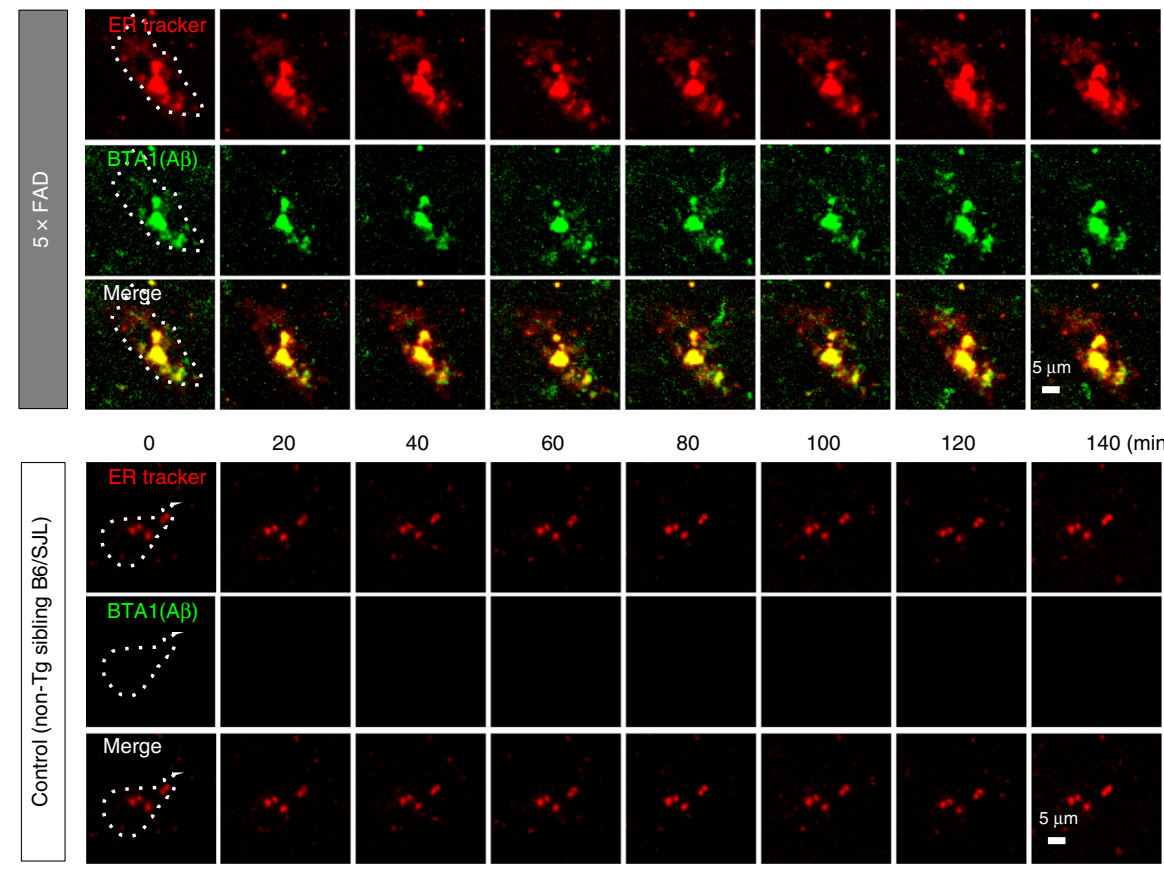

60

80

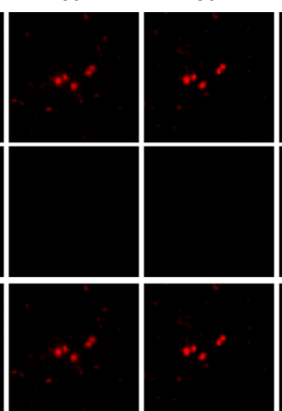

100

120

$140(\min )$

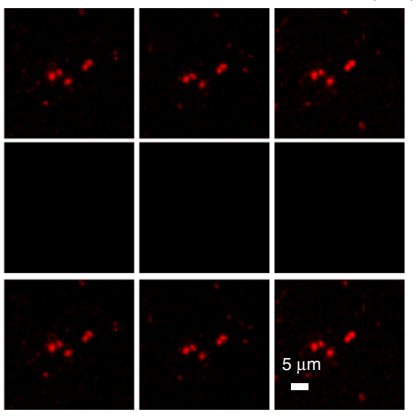

b
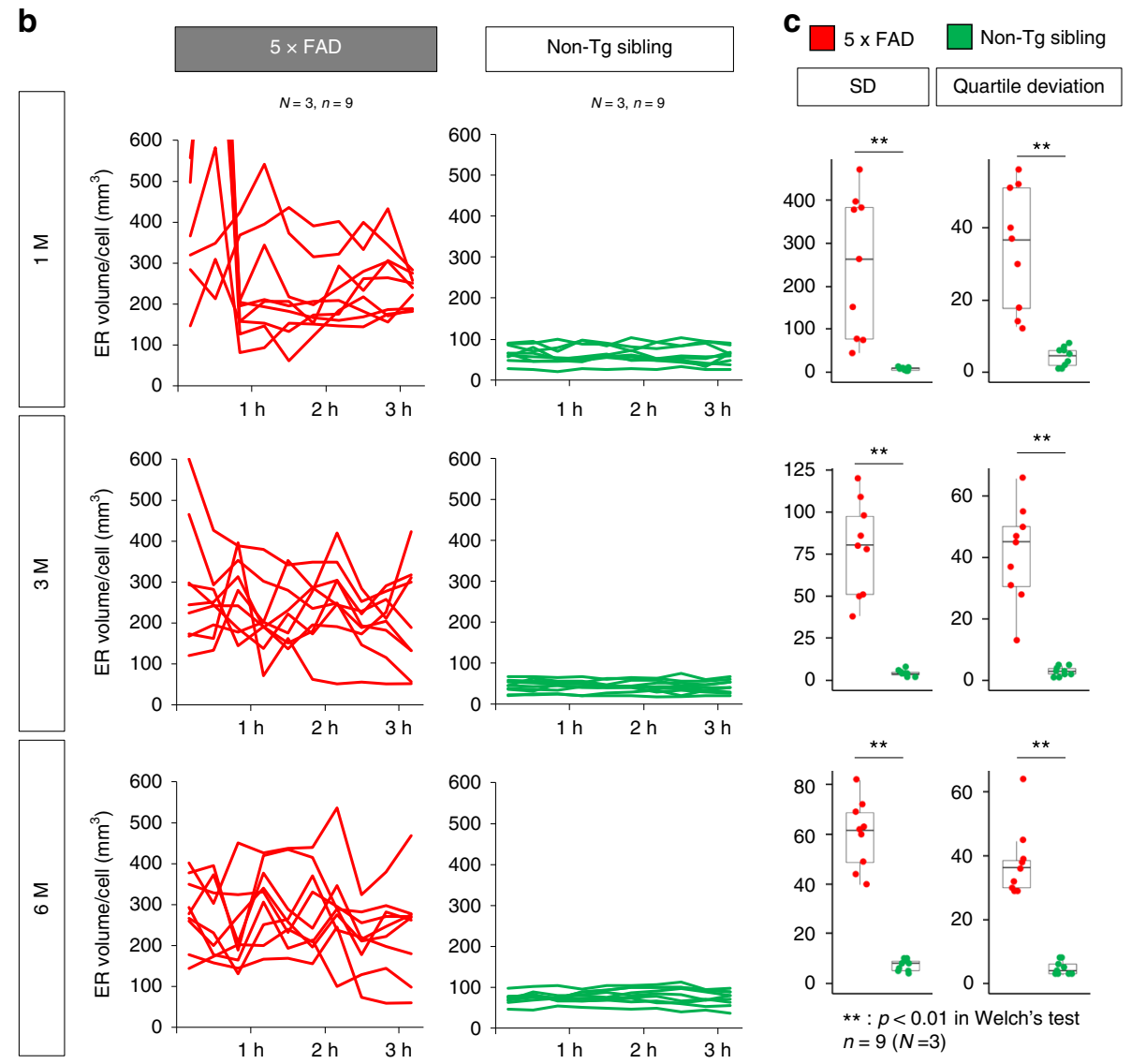

pathologically diagnosed as pure $\mathrm{AD}$ (Fig. $5 \mathrm{c}$ ) also supported the interaction between YAP and $A \beta$. Consistent with the reduced nuclear YAP in immunohistochemistry, western blot revealed a similar decrease of YAP in temporal and occipital tip tissues from $A D$ patients (Fig. 5d). YAP and $A \beta$ levels were inversely correlated in cortical tissues from the occipital tip and temporal tip of AD patients and non-neurological disease controls (Fig. 5e).

In addition, LATS1 kinase, which prevents nuclear translocation of YAP ${ }^{35}$, was activated in human cortical neurons of MCI and in those of $\mathrm{AD}$ to a lesser extent (Supplementary Fig. 9a, b). 
Fig. 3 Extreme instability of ER in AD model mice revealed by in vivo ER imaging. a In vivo ER and A $\beta$ images were acquired by two-photon microscopy from 1-month-old 5xFAD mice into which ER-tracker and BTA1 had been injected in one shot $4 \mathrm{~h}$ before observation. ER and A $\beta$ image sets were taken in tandem every $20 \mathrm{~min}$. 3D images of ER and A $\beta$ stains were merged by IMARIS (Bitplane, Zurich, Switzerland). Dot-line indicates a single neuron. $\mathbf{b}$ Total volumes of ER puncta belonging to a single cell were quantified by IMARIS (Bitplane, Zurich, Switzerland), and time courses are shown in the graph. Changes were more pronounced in 5xFAD mice than in non-transgenic sibling mice (Non-Tg sibling). $N=3$ mice, $n=9$ cells. c To verify the finding in b, standard deviation (SD) and quartile deviation of ER volumes from a single cell at multiple time points were compared between groups of 5xFAD and non-transgenic sibling mice. Box plots show the median, quartiles and whiskers that represent $1.5 \times$ the interquartile range. $P$-values were determined by Welch's test, ${ }^{\star \star} p<0.01(N=3$ mice, $n=9$ cells). Source data are provided as a "Source Data file".

On the other hand, PLK1, which switches necrosis to apoptosis ${ }^{19}$, was not activated in either MCI or symptomatic AD (Supplementary Fig. 10a, b).

The decrease of nuclear YAP due to cytoplasmic co-segregation with $\mathrm{A} \beta$ was confirmed in cortical neurons of $5 \mathrm{xFAD}$ mice (Supplementary Fig. 11a, b) and human mutant APP-KI mice (Supplementary Fig. 11c, d) at 3 months, prior to the onset of cognitive impairment. In these neurons DAPI signal intensities were decreased (Supplementary Fig. 11b, d). LATS1 activation in cortical neurons was also confirmed by immunohistochemistry in both mouse strains (Supplementary Fig. 9c, d). These findings support that Hippo pathway-dependent necrosis (TRIAD) $)^{17-19}$ occurs from the pre-symptomatic to post-symptomatic stages in both human and mouse AD pathology.

Moreover, essential transducers of necroptosis, RIP1/3 and the downstream pathways were not activated in the pathway analysis based on comprehensive phosphoproteome data (Supplementary Fig. 12a), in western blot (Supplementary Fig. 12b) and immunohistochemistry (Supplementary Fig. 12c) of cerebral cortex tissues of $5 x F A D$ mice from 1 to 3 months when YAPdependent necrosis occurred at high frequencies.

These results further support that the necrosis at the early stage of $\mathrm{AD}$ pathology is distinct from necroptosis, which had been implicated in neuronal loss at the late stage after extracellular $A \beta$ aggregation $^{36}$. In human postmortem brain of MCI due to AD, RIP1/3 were not also activated in cortical neurons possessing intracellular $A \beta$ (Supplementary Fig. 12d).

YAP deprivation by intracellular $A \beta$ induces Hippo pathwaydependent necrosis. To further uncover the mechanism of intracellular $A \beta$ induced-necrosis, we employed human induced pluripotent stem cells (iPSCs) carrying heterozygous or homozygous APP mutations (KM670/671NL) generated by genome editing ${ }^{37}$, differentiated them into neurons, and performed timelapse imaging to elucidate the chronological relationship among amount of intracellular $\mathrm{A} \beta$, transcriptional activity of TEADYAP, and ER ballooning. ER ballooning and rupture in heterozygous and homozygous AD-iPSC-derived neurons occurred at a higher frequency than in normal iPSC-derived neurons (Fig. 6a, b, Supplementary Movies 1-3), consistently with the observation in vivo (Fig. 3, Supplementary Fig. 8) and TRIAD ${ }^{17-19}$.

Interestingly, BTA1 signals reflecting intracellular A $\beta$ were increased nearly $10 \mathrm{~h}$ before initiation of ER ballooning (Fig. 6c). In addition, a TEAD-reporter vector ${ }^{38}$, which is composed of the TEAD-responsive element flanked to mCherry gene to monitor YAP co-transcriptional activity and the CMV-promoter flanked to EGFP to detect transfected cells (Fig. 6d), revealed that TEADYAP transcriptional activity was declined $8 \mathrm{~h}$ before ER ballooning in accordance with the increase of BTA1-stained intracellular $\mathrm{A} \beta$ (Fig. 6e).

We also confirmed that siRNA-mediated knockdown of YAP (Fig. 6f) directly induced ER ballooning in human normal iPSCderived neurons (Fig. 6g). Since YAP-siRNA decreased YAP protein in immunocytochemistry (Fig. 6h) and western blot (Fig. 6i) at the time point of $0 \mathrm{~min}$, the duration from the decrease of TEAD/YAP transcriptional activity to the initiation of ER ballooning was estimated to be $2-4 \mathrm{~h}$. YAP-siRNA significantly increased HMGB1 released from necrotic iPSC-derived neurons (Fig. 6j).

Injection of YAP-siRNA into cerebral cortex of normal control mice (B6/SJL) promptly induced ER instability of transfected cortical neurons under in vivo imaging by two-photon microscopy (Fig. 7a, b). Knockdown of YAP protein in siRNAtransfected neurons was confirmed in immunohistochemistry (Fig. 7c) and western blot analyses of cortex tissues (Fig. 7d). Intriguingly, we found patchy stains of pSer46-MARCKS (Fig. 7e) induced by HMGB1, a DAMPs molecule released from necrotic cells $^{23,24}$. A high magnification of such a patchy stain revealed a single or few YAP-siRNA-transfected cells with extremely weak stains of DAPI surrounded by pSer46-MARCKS (Fig. 7e) that matched well with the criteria of nuclear morphology to define active necrosis. Decreased nuclear volume revealed by quantitative analysis with $30 \mu \mathrm{m}$ sections of cortex tissues after YAPknockdown also supported TRIAD necrosis (Fig. 7f).

Moreover, we observed the whole processes from $\mathrm{A} \beta$ accumulation to ER ballooning via repression of TEAD-YAP transcriptional activity in a single iPSC-derived neuron with heterozygous or homozygous AD mutations (APP KM670/ 671NL) (Fig. 8a). EGFP-YAPdeltaC61, the neuronal isoform YAP that has the similar dynamics and roles to full-length YAP in TRIAD $^{19}$, was electroporated into neurospheres and differentiated into neurons. During the process, EGFP-YAPdeltaC61 was cosegregated to cytoplasmic $\mathrm{A} \beta$ (Fig. 8b, magenta arrow), and deprived from the nucleus (Fig. 8 b). On ER ballooning, YAP was further shifted to the ER ballooning protrusion (Fig. 8b, green arrow) and released by rupture (Fig. 8b, white arrow), while cytoplasmic A $\beta$ remained as aggregates (Fig. 8b, blue arrow). All the processes are also shown in movie (Supplementary Movies 46). We quantitatively confirmed in each neuron that the increase of BTA1 signal intensity was followed by the decrease of YAPdeltaC in the nucleus (Fig. 8c, Supplementary Movies 4-6).

Moreover, immunohistochemistry with anti-YAP and anticalnexin antibodies revealed ER enlargement in YAP-deficient neurons of human MCI patients (Fig. 8d). Consistently with iPSC-derived neuron carrying APP mutations (Fig. 8b), YAP was aggregated in the cytoplasm or translocated into ER ballooning (Fig. 8d). The similar ER ballooning was also observed in postmortem human brains of $\mathrm{AD}$ patients but at a lower frequency (Fig. 8d).

Timelapse imaging by two-photon microscopy revealed that a small part of neurons possessing intracellular $A \beta$ underwent TRIAD necrosis and the residual intracellular $A \beta$ after neuronal rupture might become seed for extracellular $A \beta$ aggregation (Supplementary Fig. 13). Since observation of the ER rupture in vivo was far more difficult technically, we could not detect the whole processes in a single neuron in vivo. However, these data in vivo and in vitro collectively suggested the sequential pathological processes of intracellular accumulation of $A \beta$, deprivation of YAP from the nucleus linked with suppression of TEAD-YAP transcriptional activity, and ER ballooning. 
a
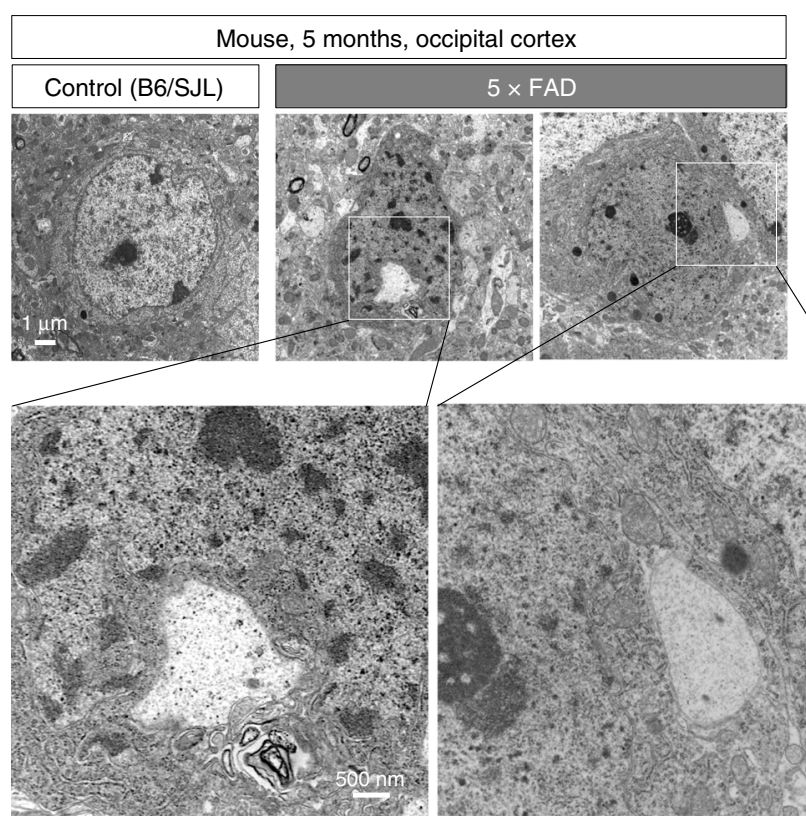

d

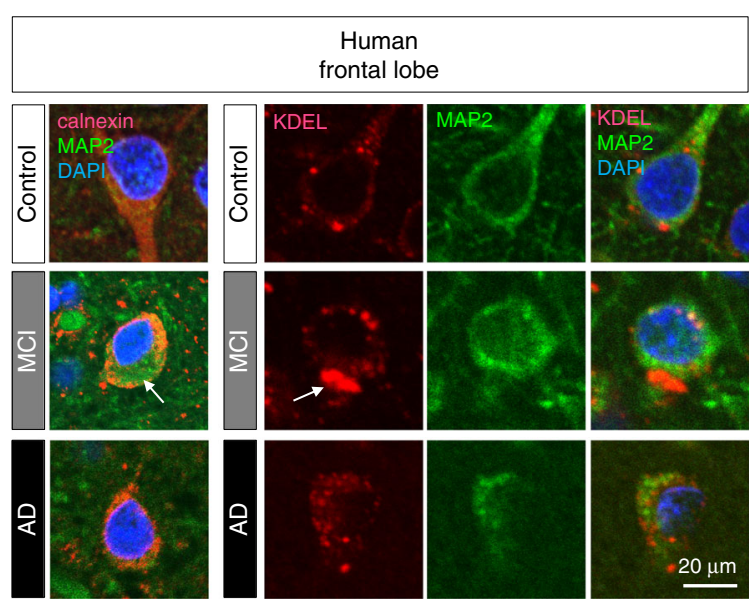

e
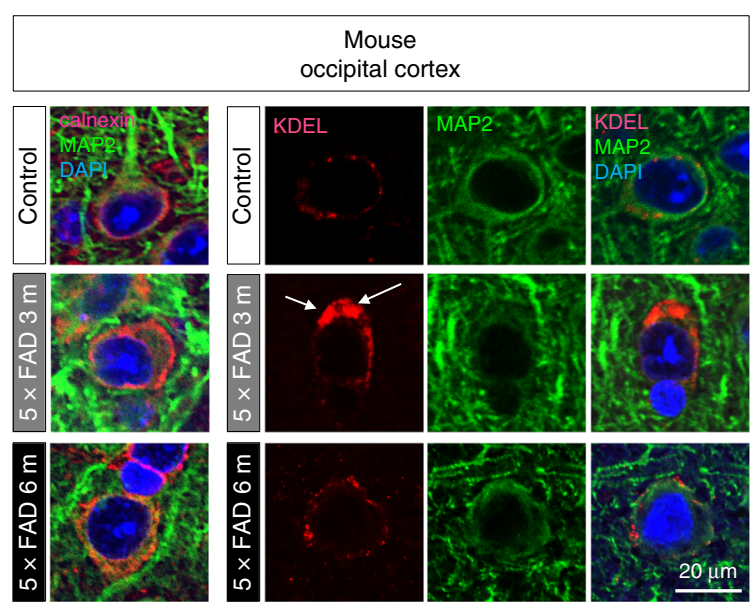

b
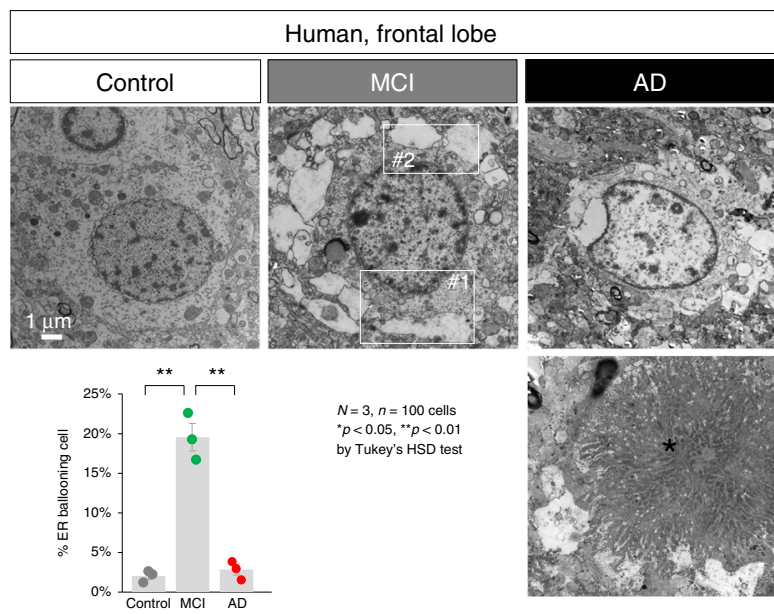

$N=3, n=100$ cells
$* p<0.05,{ }^{* *} p<0.01$

by Tukey's HSD test
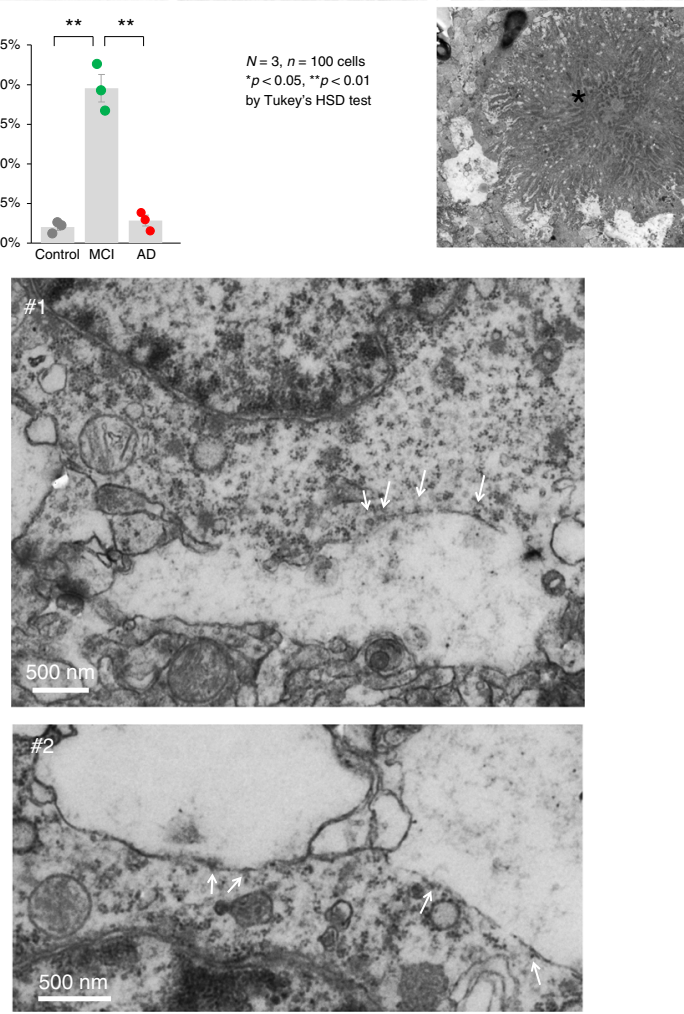

C
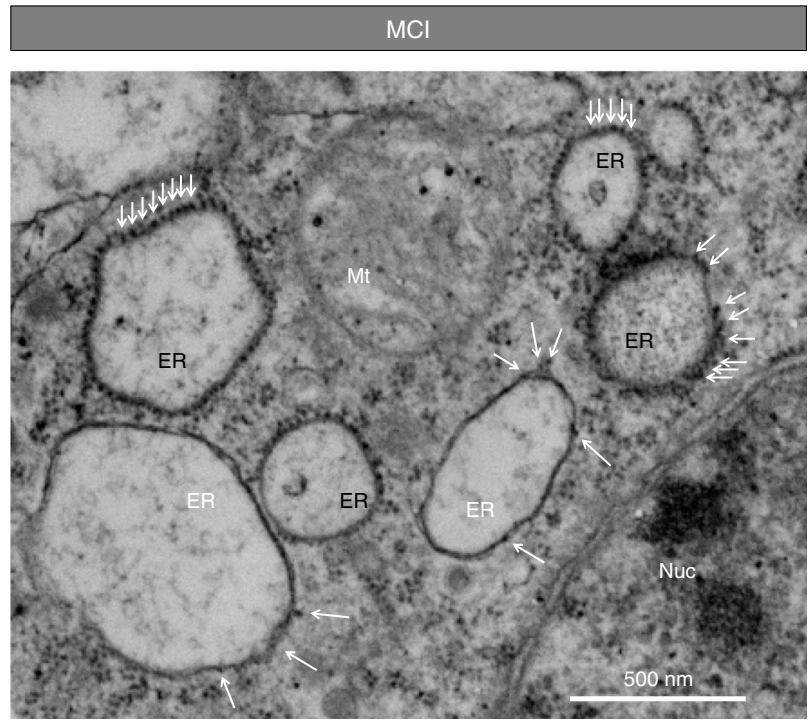

rescue the pathology of $5 x F A D$ mice. Regarding $\mathrm{S} 1 \mathrm{P}$, continuous intrathecal administration $(40 \mathrm{nM}, 0.15 \mu \mathrm{L} / \mathrm{h})$ into the CSF space was initiated at either 1 or 5 months and continued until 6 months (Fig. 9a). Regarding AAV-YAPdeltaC, one-shot injec-

tion $\left(1 \times 10^{10}\right.$ vector genomes $\left./ \mathrm{mL} \times 1 \mu \mathrm{L}\right)$ into the CSF space
S1P and YAPdeltaC rescue ER instability, necrosis, and cognitive impairment in vivo. Next, we investigated whether sphingosine-1-phosphate (S1P) and YAPdeltaC61, a neuronspecific isoform of YAP possessing a similar rescue effect to that of full-length YAP on Hippo pathway-dependent necrosis ${ }^{17-19}$, 
Fig. 4 ER enlargement of neurons in 5xFAD mice and human MCI/AD patients. a Electron microscopy of neurons in control (B6/SJL) and 5xFAD mice. Marked ER enlargement was observed in 5xFAD mice at 5 months of age before the onset. $\mathbf{b}$ Electron microscopy of neurons in non-neurological disease control, $\mathrm{MCl}$ (Braak stage III by Gallyas-Braak staining) and AD (Braak stage V) patients. Higher magnification in two subcellular fields reveal ribosomes attached to vacuoles (arrow). Amyloid plaque in AD patient is shown (asterisk). Quantitative analysis of three groups is shown in the graph. The bar graph indicates average and mean \pm S.E.M., together with the corresponding data points. P-values were determined by Tukey's HSD test, ${ }^{\star \star} p<0.01(N=3$ persons, $n=100$ cells). c A high magnification image of ballooned ER. Ribosomes on ER membrane help identification of the origin of the medium-size ERs (black ER), while ribosomes were detached when ER lumen was further enlarged and only a few ribosomes were remained (white ER). Mt: mitochondria. Nuc: nucleus. d Calnexin and MAP2 or KDEL and MAP2 co-staining of human neurons of non-neurological disease (control), MCI and AD patients. Remarkable enlargement of ER was detected (arrow) in $\mathrm{MCI}$ more frequently than in AD. e Calnexin and MAP2 or KDEL and MAP2 co-staining of mouse neurons of 5 xFAD mice at 3 and 6 months of age. Remarkable enlargement of ER was detected (arrow) at 3 months more frequently than at 6 months. Source data are provided as a "Source Data file".

between the dura and brain parenchyma was performed at 1 or 5 months, and the same series of examinations was performed (Fig. 9a). When administered from or at 1 month, S1P and AAVYAPdeltaC restored the alteration rate in the Y-maze test in $5 x F A D$ mice (Fig. 9b), although their therapeutic effects were somewhat smaller by administration from or at 5 months (Fig. 9b).

Consistently, two-photon microscopy revealed stabilization of ER volume in 5xFAD mice by S1P and AAV-YAPdeltaC (Fig. 9c). Immunohistochemistry revealed that S1P and AAV-YAPdeltaC decreased the extracellular $A \beta$ burden (Fig. 9d) in addition to the increase of nuclear YAP/YAPdeltaC (Fig. 9d). The decrease in the abundance of extracellular $A \beta$ plaques (Fig. 9d) was further confirmed by western blot (Fig. 9e) and ELISA (Fig. 9f). YAPdeltaC and total YAP were increased after the S1P and AAV-YAPdeltaC treatments in cortex tissues by western blot (Fig. 9g) and in cortical neurons by immunohistochemistry (Fig. 9h). The decrease of extracellular $A \beta$ aggregation could be explained by assuming that intracellular $A \beta$ accumulation serves as a seed for extracellular $A \beta$ aggregation after cell death ${ }^{22,39,40}$ However, further investigation is necessary to elucidate the relationship between intracellular $A \beta$ accumulation and extracellular $A \beta$ aggregation, as well as the relationship between $A \beta$ metabolism and the Hippo pathway. Given that S1P and AAVYAPdeltaC inhibit necrosis by increasing the effector molecule $Y A P$, it is clear why intracellular $A \beta$ levels were unchanged despite a reduction in necrosis (Fig. 9d). Normal sibling mice (B6/ SJL) after the similar treatments of S1P and AAV-YAPdeltaC were also examined for YAP expression, intracellular $A \beta$ and extracellular A $\beta$ levels (Supplementary Fig. 14).

S1P and YAPdeltaC rescue ER instability in AD-iPScell-derived neurons. To further evaluate S1P and AAVYAPdeltaC as a candidate therapeutic strategy in human $\mathrm{AD}$, we employed human iPSCs-derived neurons carrying heterozygous or homozygous AD mutations (APP KM670/671NL) introduced by genome editing (Fig. 10a). As mentioned in previous experiments (Fig. 6), we detected ER ballooning and rupture occurred in heterozygous and homozygous AD-iPSC-derived neurons (Fig. 10b; Supplementary Movies 1-3). BTA1 barely stained normal iPSC-derived neurons, but stained $>75 \%$ of $\mathrm{AD}$ iPSC-derived neurons, reflecting intracellular A $\beta$ accumulation (Fig. 10b, c). The frequency of ER ballooning and resultant cell death identical to the TRIAD reported in Huntington's disease ${ }^{17}$ were obviously higher in non-treated AD-iPSC-derived neurons than in non-treated normal iPSC-derived neurons (Fig. 10b, d). AD-iPSC-derived neurons accumulating intracellular $A \beta$ underwent TRIAD at a higher frequency as aforementioned (Fig. 10d, e; Supplementary Movies 7-9). As expected, $20 \mathrm{nM}$ S1P did not affect intracellular $A \beta$ accumulation (Fig. 10c) but significantly suppressed the frequency of ER ballooning and resultant cell death both in total neurons and in neurons with intracellular $A \beta$ accumulation (Fig. 10d, e; Supplementary Movies 10-12).

Similarly, we tested the effect of AAV-YAPdeltaC on ER ballooning (Fig. 10f). In this independent experiment, BTA1 stained $>75 \%$ of AD-iPSC-derived neurons (Fig. 10g). AAVYAPdeltaC remarkably suppressed the frequency of ER ballooning and resultant cell death in AD-iPSC-derived neurons (Fig. 10g, h, i, j; Supplementary Movies 13-18). We also confirmed that S1P increased the level of nuclear YAP protein (Supplementary Fig. 15a), and that AAV-YAPdeltaC increased the level of nuclear YAPdeltaC protein (Supplementary Fig. 15b, c) in AD-iPSCderived neurons. Interestingly, this overexpression of YAPdeltaC also restored nuclear endogenous full-length YAP (Supplementary Fig. 15d), presumably because overexpressed YAPdeltaC occupied intracellular $\mathrm{A} \beta$, enabling endogenous YAP to undergo nuclear translocation.

Consistent with this, TEAD-YAP/YAPdeltaC-mediated transcription, which was suppressed in heterozygous and homozygous AD-iPSC-derived neurons due to sequestration of YAP into intracellular A $\beta$ aggregates, was rescued by S1P or AAVYAPdeltaC, as evaluated by luciferase assay using a TEADresponsive element reporter plasmid (Fig. 10k, 1). Meanwhile, S1P and YAPdeltaC did not affect the amount of intracellular $\mathrm{A} \beta$, as determined by BTA1 (Fig. $10 \mathrm{c}, \mathrm{h}$ ) or anti-A $\beta$ antibody (Supplementary Fig. 15e, f), supporting that intracellular A $\beta$ accumulation occurs upstream of TEAD-YAP/YAPdeltaCmediated transcription, ER instability and cell death.

BTA1-mediated amyloid labeling suggested that $A \beta$ was mostly localized to the ER in AD-iPSC-derived neurons (Figs. 6, 10), consistent with the scenario outlined above. Higher magnification of BTA1-stained live neurons revealed that a small portion of A $\beta$ shifted from ER to cytosol (green arrow, Supplementary Fig. 16), which could be developed to intracellular A $\beta$ aggregates (Figs. 6, $8,10)$. Interestingly, before necrosis processes initiate, a very small part of $A \beta$ appeared to be excreted from cells as a small vesicle at several parts of the cell membrane (white arrow, Supplementary Fig. 16). Although the ER signals were weak in Zstack images, ER components were sometimes co-located at such vesicles in single-slice confocal microscopy images (white arrow, Supplementary Fig. 16). Such A $\beta$ secretion was also detected by immunocytochemistry with anti-A $\beta$ antibody after fixation (Supplementary Fig. 15e, f, right panels). These results suggested that $A \beta$ is secreted from intracellular $A \beta$-accumulating neurons by the exosome pathway via multivesicular bodies (MVBs).

Other types of cell death in mouse AD models and human AD patients. Finally, we summarize our data about co-existence of other types of cell death in the brains of $\mathrm{AD}$ model mice and human MCI/AD patients. Though a previous paper ${ }^{36}$ suggested increased necroptosis in postmortem human AD brains, they used antibodies against non-phosphorylated RIP1/3 and did not show co-activation of RIP1/3 and MLKL. It is not 
a

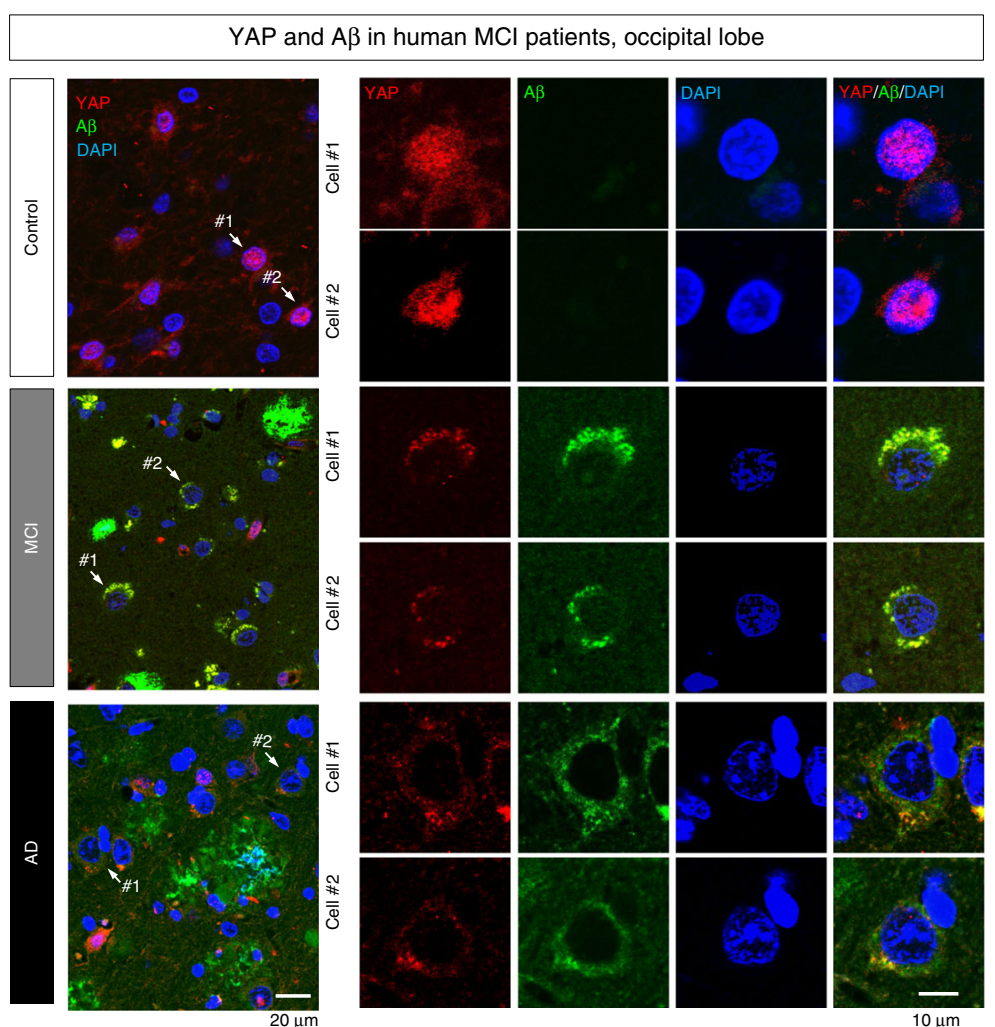

d

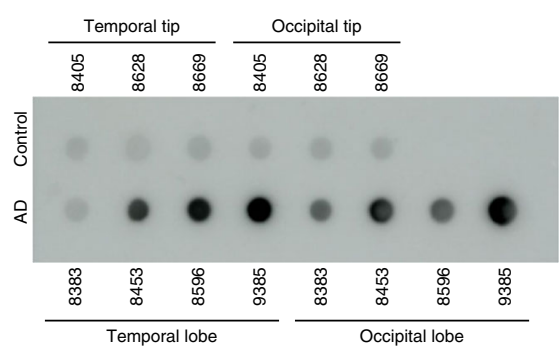

$$
\text { Control }
$$

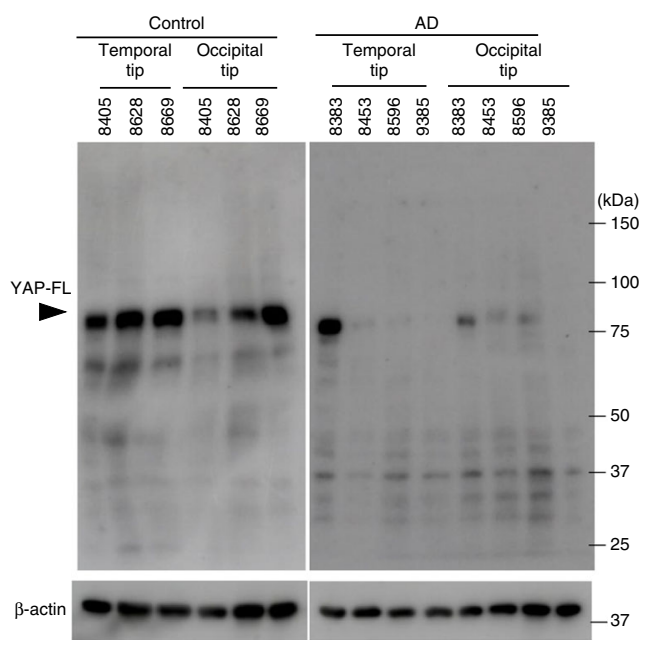

b
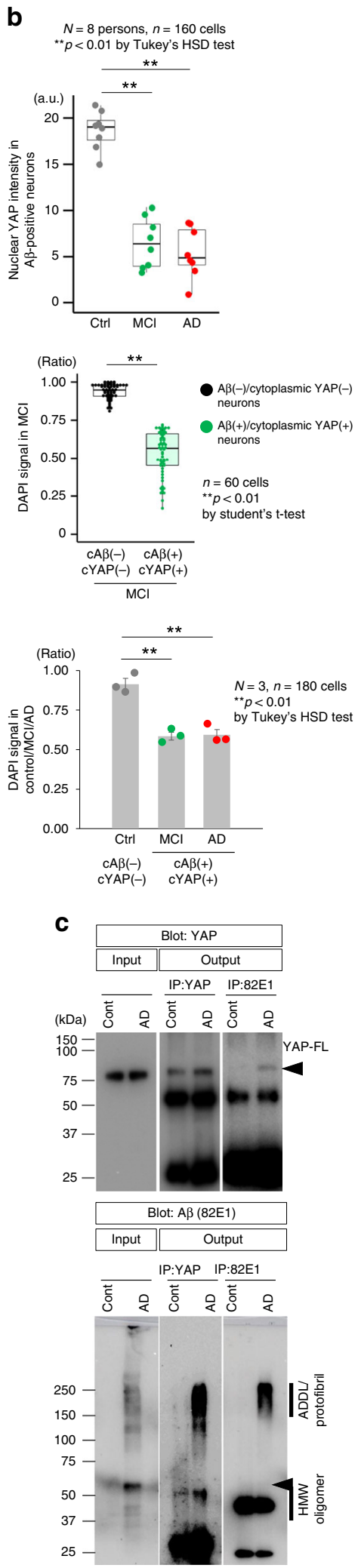

non-phosphorylated RIP1/3 and non-phosphorylated MLKL but phosphorylated RIP1/3 and phosphorylated MLKL that forms the signal transducing complex necrosome executing necropto$\operatorname{sis}^{41-44}$. In our immunohistochemistry of pRIP1/3 and pMLKL, with brain samples of $\mathrm{AD}$ model mouse and human $\mathrm{AD}$ patient,

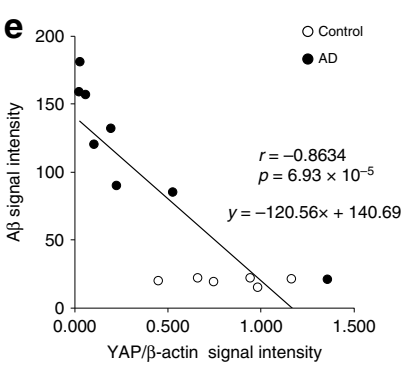

we could not detect co-localization of phosphorylated RIP1/3 and phosphorylated MLKL, which is essential for signal transduction of necroptosis, in any single neuron of $5 \mathrm{xFAD}$ or APP-KI mice at 3 months of age or of human MCI and AD patient (Supplementary Fig. 17). In parallel experiments of cerebral cortex tissues 
Fig. 5 A $\beta$ sequesters YAP from the nucleus to intracellular aggregates. a Immunohistochemistry of YAP and A $\beta$ of human postmortem brains revealed sequestration of YAP into cytoplasmic $A \beta$ aggregates and the resultant decrease of nuclear YAP in $\mathrm{MCl}$ and $A D$ patients. $\mathbf{b} Q$ Quantification of signal intensities of nuclear YAP staining in neurons confirmed similar findings in three $\mathrm{MCl}$ patients and three symptomatic $A D$ patients. $A$ total of 160 neurons ( $N=8$ persons $)$ in the occipital lobe were selected at random from each patient, and nuclear YAP signal intensities were quantified by confocal microscopy (FV1200IXGP44, Olympus, Tokyo, Japan). Signal intensity of DAPI per a nucleus was quantified in cortical neurons of occipital lobe of non-neurological disease controls and $\mathrm{MCl}$ or $\mathrm{AD}$ patients. The DAPI signals were compared in $\mathrm{MCl}$ between cytoplasmic YAP-positive and A $\beta$-positive neurons ( $n=60$ ) and cytoplasmic YAP-negative and A $\beta$-negative neurons $(n=60)$. In addition, signal intensity of DAPI per a nucleus of cytoplasmic was compared between YAP-positive and A $\beta$-positive neurons $(n=180)$ of $\mathrm{MCl}$ or $\mathrm{AD}$ patients $(N=3)$ and normal neurons $(n=180)$ of non-neurological disease controls $(N=3)$. Box plots show the median, quartiles and whiskers that represent $1.5 \times$ the interquartile range. The bar graph indicates average and mean \pm S.E.M., together with the corresponding data points. P-values were determined by Tukey's HSD test or student's $t$-test, ${ }^{\star \star} p<0.01$ c Immunoprecipitation reveals the interaction between YAP and A $\beta$ in human postmortem brain. Upper panels: anti-A $\beta$ antibody (82E1) co-precipitated YAP from cerebral cortex tissues of AD patients, but not from non-neurological disease control. Lower panels: reverse co-precipitation of $A \beta$ protofibrils with YAP. d Western and dot blots of $A \beta$ and YAP. Temporal tip and occipital tip tissues from pathologically diagnosed AD patients or controls were immunoblotted with anti-A $\beta$ antibody (82E1, dot blot) or anti-YAP antibody (sc-15407). e Inverse correlation between A $\beta$ burden and YAP in human patient/control brains. P-values were determined by Pearson's correlation coefficient (AD: $N=8$ persons, Control: $N=6$ persons). Source data are provided as a "Source Data file".

after ischemia as a positive control of necroptosis, co-localization of pRIP1/3 and pMLKL was confirmed almost in all neurons (Supplementary Fig. 17).

In our human $\mathrm{AD}$-iPSC-derived neurons, morphological classification according to a previous report ${ }^{19}$ revealed that $<20 \%$ of neurons shrunk without cytoplasmic ballooning, mimicking apoptosis (Fig. 10m). However, the percentage of such apoptotic shrinkage or necrotic rupture was not significantly different among normal, heterozygous and homozygous APP-mutant neurons (Fig. 10n). Moreover, YAP-siRNA increased the shrinkage type of cell death while the extent of increase was not different between scrambled control siRNA and YAP-siRNA (Fig. 10o).

Collectively, these data supported that YAP-dependent TRIAD necrosis is a dominant form of cell death at the early stage of $\mathrm{AD}$ pathology, and could be a therapeutic target to cease the progression.

\section{Discussion}

Morphological detection of neurons under the dying process is difficult because the cells lose both chemical and immunohistological staining. However, a sensitive marker (pSer46-MARCKS) of degenerative neurites surrounding dying neurons enabled us to detect necrosis efficiently. This technique revealed that the frequency of necrosis reaches a peak during the preclinical stage of $\mathrm{AD}$ pathology in two types of $\mathrm{AD}$ mouse models. Moreover, the technique revealed that active necrosis is more abundant at the prodromal stage of MCI than the clinical stage of AD in human patients (Fig. 2d, e). To the best of our knowledge, only the Herrup group has investigated cell death in MCI by using cell cycle markers while their focus was other than the chronological change of cell death ${ }^{45}$.

Regarding the dynamics of active necrosis, we generated a formula based on the hypothesis that cell death occurs at a constant rate in the residual neurons and in regular time interval. Predicted number of active necrosis declined immediately after the onset of cell death, and explained well the actual chronological change (Fig. 2f, g). Moreover, multiple expected parameters also matched very well with the observed data (Fig. 2h) verifying the formula.

In addition, we determined that neuronal cell death in the early stage of $\mathrm{AD}$ is Hippo pathway-dependent necrosis, similar to that induced by RNA polymerase II inhibitor ${ }^{17,18}$ or YAP sequestration by mutant $\mathrm{Htt}^{19}$. In the case of $\mathrm{AD}$, YAP is sequestered to cytoplasmic $A \beta$, eventually impairing the function of YAP in the nucleus (Figs. 5-8). It remains unclear why YAP interacts with multiple causative proteins of neurodegenerative diseases.
However, YAP is a member of $\operatorname{IDPs}^{46}$, a family that includes TDP43, FUS, tau, $\alpha$-synuclein, and so on, which mutually interact and are involved in neurodegenerative diseases ${ }^{47}$. We found that low-complexity sequences are distributed throughout mouse and human YAP (Supplementary Fig. 18), supporting that full-length YAP and YAPdeltaC could interact with $A \beta$ via intrinsically denatured regions.

Interestingly, a recent study implicated YAP as a hub molecule in $\mathrm{AD}$ pathology ${ }^{10}$. Xu and colleagues performed a meta-analysis of functional genomic data of $\mathrm{AD}$ and concluded that YAP is the most important hub molecule in the molecular network of $\mathrm{AD}^{10}$. Their subsequent experiments showed that YAP-KD increased the levels of $A \beta^{10}$, consistent with our results. Thus, the increase in the YAP mRNA level that they observed ${ }^{10}$ could represent a protective transcriptional response aimed at compensating for the reduced level of YAP protein. Although their results did not reveal the direct relevance of YAP to neuronal cell death in $\mathrm{AD}$, their findings match very closely with our observations and hypothesis.

Although cell death has been generally suspected as a terminalstage pathology in $\mathrm{AD}$, the evidence in support of this idea remains weak. Our experimental results suggest an alternative view regarding the timing and roles of cell death in AD (Supplementary Fig. 19). Intriguingly, Hippo pathway-dependent TRIAD necrosis occurs at an early stage and plays some critical roles in the progression of AD pathology. First, as a cellautonomous process of degeneration, intracellular $A \beta$-induced necrosis decreases the number of cerebral neurons via sequestration of YAP. Second, as a non-cell-autonomous process necrotic neurons release alarmins/DAMPs that trigger secondary cell damage in surrounding neurons. This process could expand degeneration to bystander neurons that contain only a low level of intracellular $A \beta$. Third, after necrosis, intracellular $A \beta$ becomes the seed for extracellular $A \beta$ aggregation, representing another non-cell-autonomous means of expanding degeneration. Fourth, prionoid transmission of $A \beta$ and tau proteins could be also promoted by TRIAD necrosis, as shown by live images of $\mathrm{AD}$ iPSC-derived neurons (Supplementary Figs. 15, 16).

Restoration of the YAP protein level using an AAV vector successfully inhibited necrosis during the early stage of $\mathrm{AD}$. More importantly, the treatment efficiently prevented cognitive impairment and extracellular $\mathrm{A} \beta$ aggregation in $\mathrm{AD}$ model mice. Paired experiments using AD-iPSC-derived neurons further supported the therapeutic effects of YAPdeltaC. Given that no extracellular $\mathrm{A} \beta$ aggregates existed under our culture condition, the experiment directly indicated that the necrosis was not 
a

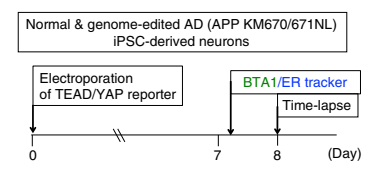

b

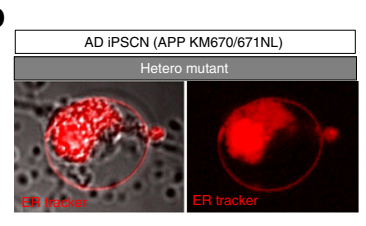

C

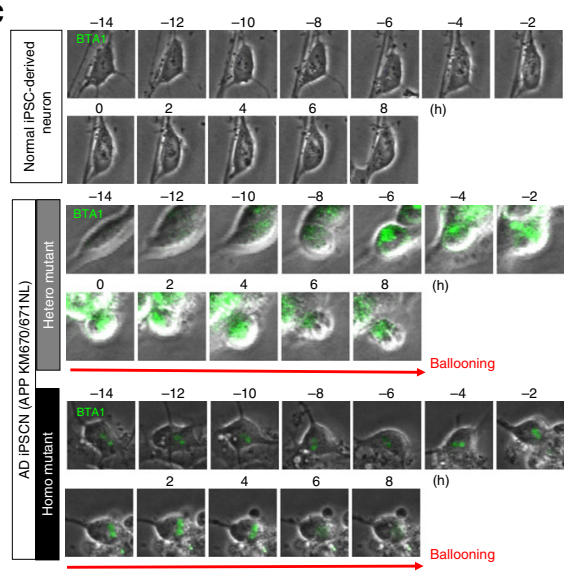

d
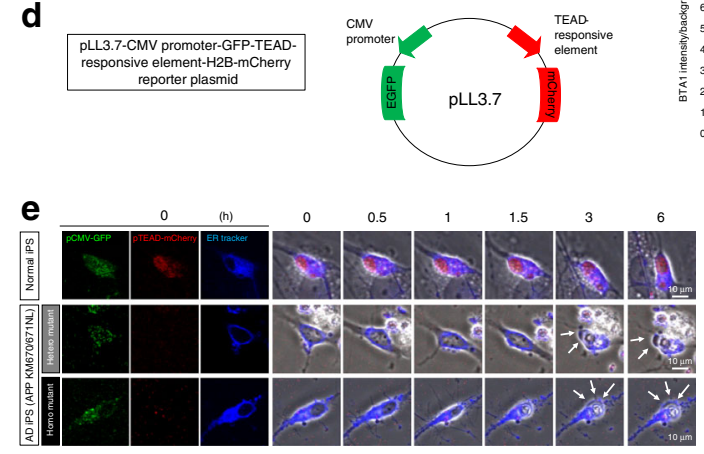
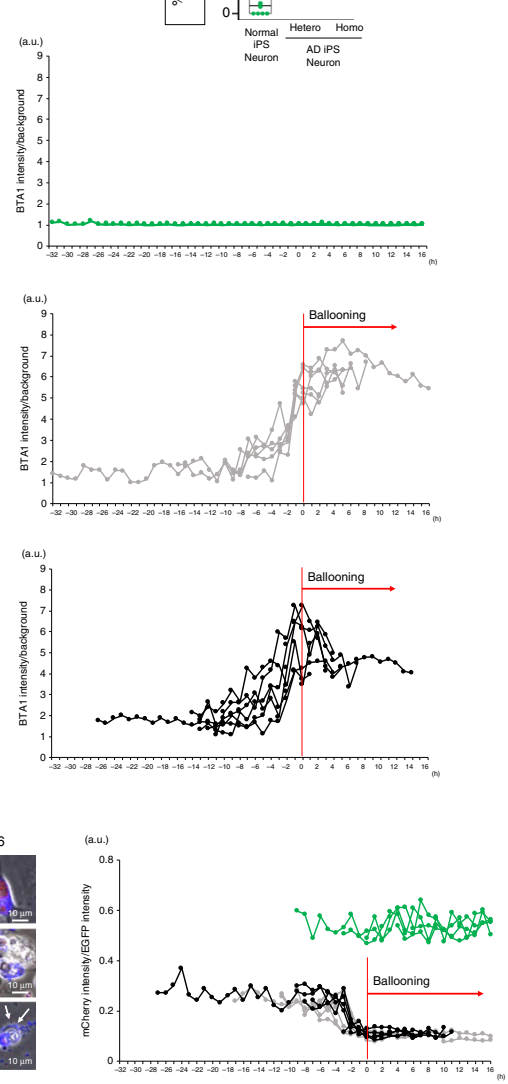

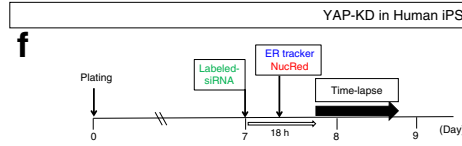

g
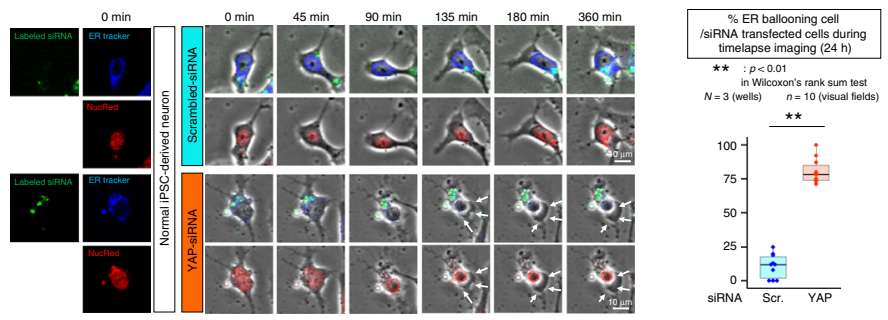

timelapse imaging (24 h)

in Wilcoxon's rank sum test
$N=3$ (wells)
$n=10$ (visual filds)

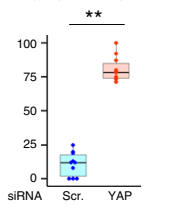

h
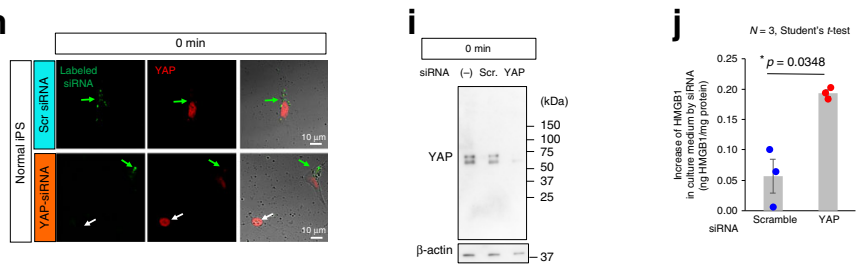

derived from extracellular $A \beta$ aggregation but from intracellular $\mathrm{A} \beta$ accumulation. Early-stage intervention in molecules regulating Hippo pathway-dependent necrosis, or in triggering of necrosis by intracellular $A \beta$, could suppress progression to the late-stage pathological changes, possibly including extracellular
A $\beta$ aggregation. Long-term follow-up of AAV-YAPdeltaCtreated mice for up to 6 months did not reveal tumors in systemic organs or the brain. However, further investigation at the GMP level vector would be necessary to finally confirm the safety of AAV-YAPdeltaC as a human therapeutic vector. 
Fig. 6 Time lapse imaging of multiple neurons suggests pathological cascade. a Experimental protocol to evaluate the relationship among intracellular $A \beta, T E A D / Y A P$ transcriptional activity and ER ballooning. $\mathbf{b}$ High magnification revealed that the membrane of the cytoplasmic balloon was reactive to ERtracker in iPSC-derived neurons carrying APP mutations (left panels). ER ballooning occurs frequently in iPSC-derived neurons carrying APP mutations (heterozygous and homozygous mutants carrying APP KM670/671NL) (right graph). P-values were determined by Wilcoxon's rank sum test with posthoc Bonferroni correction, ${ }^{\star \star} p<0.01$ ( $N=3$ wells, $n=10$ visual fields). c Accumulation of BTA1-stained intracellular A $\beta$ occurs before ER ballooning in iPSC-derived neurons carrying APP mutations (left panels). Alignment of BTA1 signals changes in multiple neurons to the time point of ER ballooning initiation revealed intracellular $A \beta$ began to accumulate $10 \mathrm{~h}$ before ER ballooning (right graphs). $\mathbf{d}$ Construction of a plasmid vector to monitor TEAD/YAPtranscriptional activity. e TEAD/YAP transcriptional activity was decreased in iPSC-derived neurons carrying APP mutations in comparison to normal iPSCderived neurons (left panels). Alignment to ER ballooning time point revealed TEAD/YAP transcriptional activity started to decrease $8 \mathrm{~h}$ before ER ballooning. These results suggest a pathological cascade, increase of intracellular A $\beta \rightarrow$ decrease of TEAD/YAP transcriptional activity $\rightarrow$ ER ballooning. f Protocol of YAP-knockdown in normal human iPSC-derived neurons. $\mathbf{g}$ Time lapse imaging of siRNA-transfected neurons revealed the increase of ER ballooning by YAP-siRNA. Right graph shows quantitative analysis. P-values were determined by Wilcoxon's rank sum test, ${ }^{\star \star} p<0.01(\mathrm{~N}=3$ wells, $n=10$ visual fields). $\mathbf{h}$ Conformation of siRNA-mediated YAP-knockdown by immunohistochemistry. Green and white arrows indicate siRNA-mediated and nontransfected cells, respectively. $\mathbf{i}$ Conformation of siRNA-mediated YAP-knockdown by western blot. $\mathbf{j}$ HMGB1 concentration in culture medium was quantified by using ELISA, and the increase of HMGB1 from the initial concentration after siRNA transfection was compared between scrambled control siRNA and YAP-siRNA. $P$-values were determined by Student's $t$-test $(N=3$ wells $)$. The box plot shows the median, quartiles and whiskers that represent $1.5 \times$ the interquartile range. The bar graph indicates average and mean \pm S.E.M., together with the corresponding data points. Source data are provided as a "Source Data file".

Type III cell death with cytoplasmic changes in human AD brains that is homologous to TRIAD has been repetitively described in old historical papers of neuropathology. For instance, Hirano and colleagues ${ }^{48}$ reported granulovacuolar body, which is a homologous large vacuole found in pyramidal neurons in Sommer's sector of senile dementia, AD and Pick's disease (now a form of FTLD). Another examples is the paper by Dickson and colleagues ${ }^{49}$, which described ballooned neurons in $\mathrm{AD}$, Pick's disease, cortico-nigral degenration, and pigment-spheroid degeneration. Our previous work revealed TRIAD in Huntington's disease pathology ${ }^{50}$, a number of papers have reported a cell death morphologically homologous to TRIAD ${ }^{51-56}$.

The current definition of MCI is largely based on subjective complaints by patients who have insufficient cognitive decline to be diagnosed with dementia and who remain adequately socially adjusted. No objective markers are available to support the subjective diagnosis or to evaluate the pathological state during MCI stage. Therefore, in combination with amyloid PET to quantify the extracellular A $\beta$ burden, the use of CSF-HMGB1 to detect the amount of on-going cell death could serve as a sensitive quantitative marker for evaluating disease progression and also the effect of candidate drugs.

In conclusion, we have provided evidence that neuronal necrosis induced by YAP deprivation occurs most actively in the early stages of $\mathrm{AD}$, including preclinical $\mathrm{AD}, \mathrm{MCI}$ or ultra-early stage of $\mathrm{AD}$ before extracellular $\mathrm{A} \beta$ aggregation. In addition, we showed that CSF-HMGB1 is a powerful tool for evaluating the activity of cell death in such stages. We also proposed therapeutic approaches targeting the change in the level of nuclear YAP in neurons, i.e., targeting the Hippo pathway-dependent necrosis.

\begin{abstract}
Methods
Patient cohort. A summary of all patient information is provided in Supplementary Table 1 . Cohort 1 consists of four normal controls, one patient without dementia but with another neurological disease (disease control), 19 patients with $\mathrm{MCI}$, and 18 patients with $\mathrm{AD}$. Cohort 2 comprised 13 disease controls, seven MCI patients, and $17 \mathrm{AD}$ patients. Cohort 3 comprised 30 normal controls and $30 \mathrm{AD}$ patients. Cohort 4 comprised eight $\mathrm{AD}$ patients. Informed consent for the use of all human CSF was obtained and approved by the appropriate ethics committee at each institution and by Tokyo Medical and Dental University.
\end{abstract}

Mini-Mental State Examination. The Japanese version of the Mini-Mental State Examination (MMSE) was performed by the corresponding physician of each patient.

CSF sampling. All CSF samples were obtained by lumbar puncture before meal times and collected into polypropylene tubes. The CSF samples were centrifuged $\left(1000 \times g\right.$ for $10 \mathrm{~min}$ at $\left.4{ }^{\circ} \mathrm{C}\right)$ to remove any debris, and then stored in small aliquots at $-80^{\circ} \mathrm{C}$.

$\mathbf{A} \boldsymbol{\beta}$ and tau measurement. CSF- $A \beta 1-40$ and $-A \beta 1-42$ were measured by enzymelinked immunosorbent assay (ELISA) using a human $\beta$ amyloid (1-40) ELISA kit (292-62301, Wako Chemical Co., Saitama, Japan) and human $\beta$ amyloid (1-42) ELISA kit (298-62401, Wako Chemical Co., Saitama, Japan). CSF-pTau proteins were measured using INNOTEST Phospho-tau (181 P, Innogenetics, Ghent, Belgium).

High-sensitivity HMGB1 ELISA. Polystyrene microtiter plates (152038, Nunc, Roskilde, Denmark) were coated with $100 \mu \mathrm{L}$ of anti-human HMGB1 monoclonal antibody (1 mg/L, Shino-Test, Tokyo, Japan) in PBS and incubated overnight at $2-8{ }^{\circ} \mathrm{C}$. The plates were washed three times with PBS containing $0.05 \%$ Tween 20 , and then incubated for $2 \mathrm{~h}$ with $400 \mu \mathrm{L} /$ well PBS containing $1 \%$ BSA to block remaining binding sites. After the plates were washed again, $100 \mu \mathrm{L}$ of each dilution of the calibrator and CSF samples (1:1 dilutions in $0.2 \mathrm{M}$ Tris $\mathrm{pH} 8.5,0.15 \mathrm{M} \mathrm{NaCl}$ containing $1 \%$ BSA) was added to the wells. The plates were then incubated for $24 \mathrm{~h}$ at $37^{\circ} \mathrm{C}$. The plates were washed again, and then incubated with $100 \mu \mathrm{L} /$ well peroxidase-conjugated anti-human HMGB1,2 monoclonal antibody (Shino-Test, Tokyo, Japan) for $2 \mathrm{~h}$ at room temperature. After another washing step, the chromogenic substrate 3,3',5,5'-tetra-methylbenzidine (T022, Dojindo Laboratories, Kumamoto, Japan) was added to each well. The reaction was terminated with $0.35 \mathrm{M} \mathrm{Na}_{2} \mathrm{SO}_{4}$, and absorbance at $450 \mathrm{~nm}$ was read on a microplate reader (Model 680, Bio-Rad Laboratories, Hercules, CA, USA). A standard curve was obtained using purified pig thymus HMGB1 (Shino-Test, Tokyo, Japan). CSF samples with HMGB1 concentrations of 300 and $1000 \mathrm{pg} / \mathrm{mL}$ were analyzed to assess intra-assay $(n=10)$ and inter-assay precision $(n=10)$. The coefficients of variation in the intra- and inter-assay were $4.8-6.1 \%$ and $4.1-9.1 \%$, respectively. The working range for the assay was $100-5000 \mathrm{pg} / \mathrm{mL}$. Recovery of purified pig thymus HMGB1 added to pooled CSF was $80-105 \%(n=10)$.

Human tissue samples. Paraffin sections and frozen brain tissues were prepared from human $\mathrm{MCI} / \mathrm{AD}$ brains and disease control brains without dementia (nonneurological disease controls). Informed consent for the use of human tissue samples was obtained, after approval of the ethics committee at each institution and Tokyo Medical and Dental University.

AD model mice. 5xFAD transgenic mice overexpressing mutant human APP (770) with the Swedish (KM670/671NL), Florida (I716V), and London (V717I) familial Alzheimer's disease (FAD) mutations and human PS1 with FAD mutations (M146L and L285V) were purchased from The Jackson Laboratory (34840-JAX, Bar Harbor, ME, USA). Both the APP and PS1 transgenes were under the contro of the mouse Thy1 promoter ${ }^{33}$. The backgrounds of the mice were C57BL/SJL, which was produced by crossbreeding C57BL/6 J female and SJL/J male mice. APPKI mice possess a single human APP gene with the Swedish (KM670/671NL), Arctic (E693G), and Beyreuther/Iberian (I716F) mutations ${ }^{34}$.

Behavioral analysis. Exploratory behavior was assessed using a Y-shape maze consisting of three identical arms with equal angles between each arm (YM-3002, O'HARA \& Co., Ltd., Tokyo, Japan). Mice at the age of 2 months were placed at the end of one arm and allowed to move freely through the maze during an $8 \mathrm{~min}$ session. The percentage of spontaneous alterations (indicated as an alteration 
a

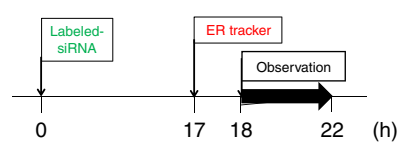

b
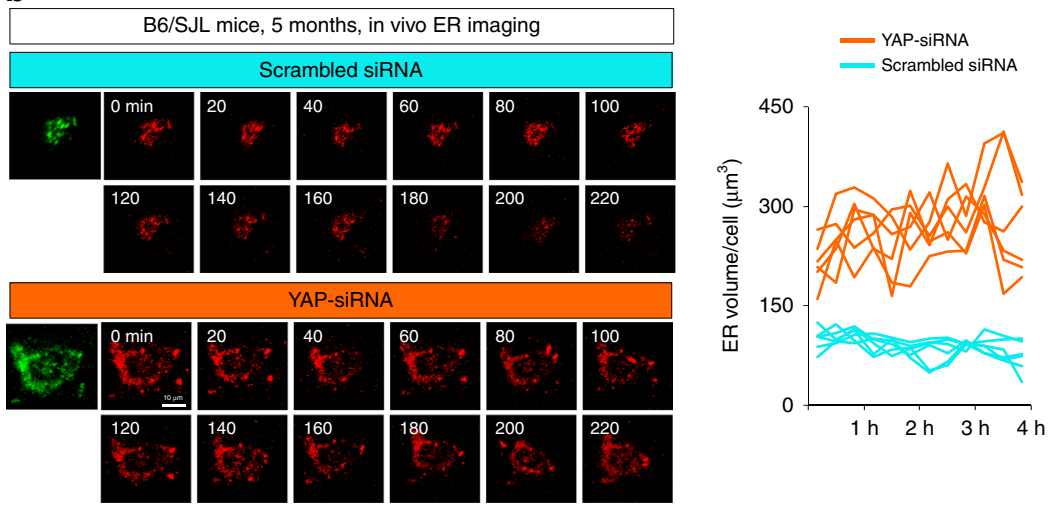

C

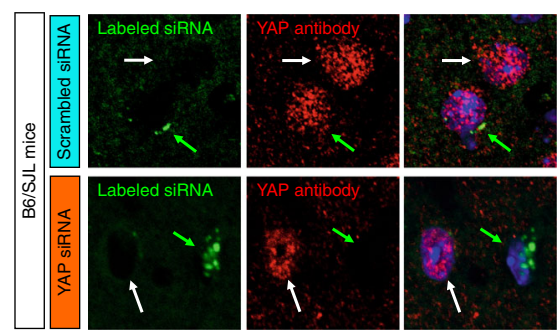

e
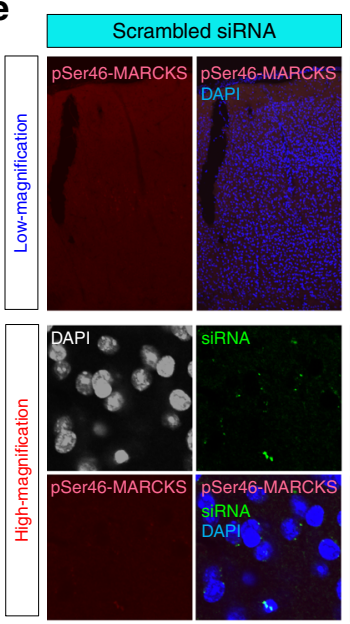

f

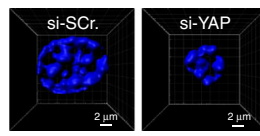

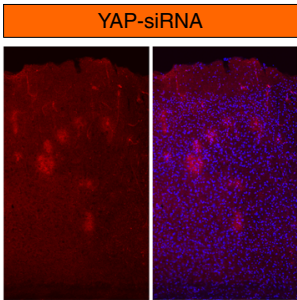
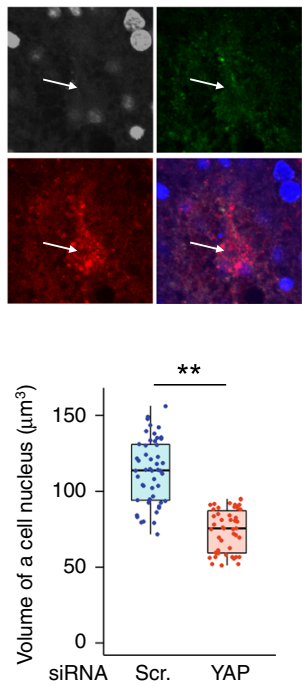

d

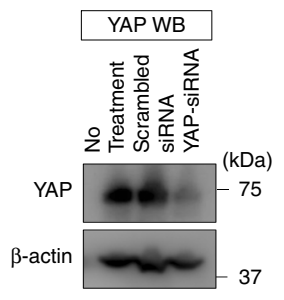

$N=3, n=30$ fields

${ }^{* *} p<0.01$

by student's $t$-test

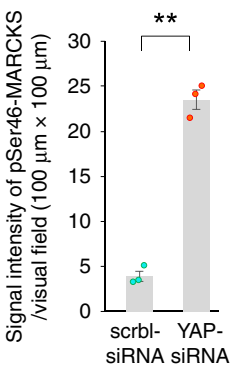

${ }^{* *}: p<0.01$

in Wilcoxon's rank sum test $N=3, n=50$ cells score) was calculated by dividing the number of entries into a new arm different from the previous one by the total number of transfers from one arm to another arm.

Re-evaluation of anti-pSer46-MARCKS antibody. Purification protocol, ELISA, and Immunohistochemistry: [ENGHVKVNGDA(pS)PA] and [ENGHVKVNG
DASPA] peptides were synthesized, added cysteine at $\mathrm{N}$ terminus, and conjugated with KLH (keyhole-limpet hemocyanin). Two rabbits were immunized eight times during nine weeks by the phospho-peptide, and the serum collected at one week after the final immunization was loaded onto non-phospho-peptide column made by [ENGHVKVNGDASPA] peptide and then onto phospho-peptide column made by [ENGHVKVNGDA(pS)PA] peptide. Antibodies bound to each column were eluted by $0.1 \mathrm{M}$ glycine-HCl buffer ( $\mathrm{pH} 2.5$ ). 
Fig. 7 YAP knockdown induces ER ballooning in normal mice. a Protocol of YAP knockdown in vivo. Labeled YAP-siRNA or scrambled control RNA was transfected into normal mice (B6/SJL), and ER images of siRNA-positive neurons were obtained by two-photon microscopy from $18 \mathrm{~h}$ later for $4 \mathrm{~h}$. $\mathbf{b}$ In vivo time-lapse imaging of normal mice after siRNA transfection by two-photon microscopy. Right graph shows quantitative analysis of ER volume of siRNApositive neurons. c Knockdown of YAP was confirmed by immunohitochemistry after in vivo imaging of two-photon microscopy. Green arrow indicates siRNA-transfected cell, and white arrow indicates non-transfected cell. d Knockdown of YAP was confirmed by western blot of mouse cortex tissues dissected after observation by two-photon microscopy. e Immunohistochemistry of pSer46-MARCKS after siRNA transfection. Patty immunostains were observed by transfection of YAP-siRNA but not scrambled control siRNA (low magnification). High magnification revealed DAPI-negative cell death surrounded by pSer46-MARCKS signals. Quantitative analysis confirmed the increase of pSer46-MARCKS immunostain signals by YAP-siRNA (right graph). The bar graph indicates average and mean \pm S.E.M., together with the corresponding data points. $P$-values were determined by Student's $t$-test, ${ }^{\star \star} p<0.01$ ( $N=3$ mice, $n=30$ fields). $\mathbf{f}$ Quantitative analysis of nuclear volume after YAP-knockdown supported TRIAD necrosis. Left panels:

representative nuclei in $3 \mathrm{D}$ imaging, right graph: quantitative analysis of a cell nucleus. The box plot shows the median, quartiles and whiskers that represent $1.5 \times$ the interquartile range. $P$-values were determined by Wilcoxon's rank sum test, ${ }^{\star \star} p<0.01(N=3$ mice, $n=50$ cells). Source data are provided as a "Source Data file".

ELISA was employed to examine the specific reactivity of anti-pSer46-MARCKS antibody to pSer46-MARCKS as follows. In $50 \mu \mathrm{L}$ of $20 \mu \mathrm{g} / \mathrm{mL}$ phosphorylated peptide [ENGHVKVNGDA(pS)PA] and non-phosphorylated peptides [ENGHV KVNGDASPA] in PBS were added to each well of the plates (Elisa Plate strips, \#FEP-100-008-1, Guangzhou JET Bio-Filtration Products, Co., Ltd, Guangzhou, China) and left for $2 \mathrm{~h}$ at room temperature. After washing three times with PBS, $200 \mu \mathrm{L}$ of $2 \%$ BSA in PBS was added to each well and incubated for $2 \mathrm{~h}$ at room temperature. After washing the plate twice with PBS, $100 \mu \mathrm{L}$ of diluted antipSer46-MARCKS antibody in PBS was added to each well of the plates and incubated $2 \mathrm{~h}$ at room temperature. After washing the wells four times with PBS, $100 \mu \mathrm{L}$ of HRP-conjugated secondary antibody (ab150077, Abcam, Cambridge, UK) diluted in PBS was added to each well of the plates and incubated $1 \mathrm{~h}$ at room temperature. After washing the plate four times with PBS, $100 \mu \mathrm{L}$ of TMB (ELTMB Chromogenic Reagent kit, C520026, Sangon Biotech, Shanghai, China) was added to start the reaction and incubated after sufficient color development, and the reaction was stopped with $100 \mu \mathrm{L}$ of $2 \mathrm{M} \mathrm{H}_{2} \mathrm{SO}_{4}$. Absorbance was measured using a plate reader (iMark, 681130J1, Bio-Rad Laboratories, Hercules, CA, USA) at $450 \mathrm{~nm}$.

Immunohitochemistry were performed as described in the following. Brain tissue sections of 6-month old 5xFAD mice were incubated with the antibodies against Ser46-phosphorylated- or non-phosphorylated-MARCKS at a dilution of 1:2000 overnight at room temperature.

Immunohistochemistry. For immunohistochemistry, mouse or human brains were fixed with $4 \%$ paraformaldehyde and embedded in paraffin. Sagittal or coronal sections (5 $\mu \mathrm{m}$ thickness) were obtained using a microtome (REM-710, Yamato Kohki Industrial Co., Ltd., Saitama, Japan). Immunohistochemistry was performed using the following primary antibodies: rabbit anti-pSer46-MARCKS (1:2000, ordered from GL Biochem Ltd., Shanghai, China); mouse anti-amyloid $\beta$ (1:5000, clone 82E1, \#10323, IBL, Gunma, Japan); rabbit anti-calnexin (1:200, ab58503, Abcam, Cambridge, UK); mouse anti-KDEL (1:100, ADI-SPA-827, Enzo Life Sciences, NY, USA); rabbit anti-MAP2 (1:200, ab32454, Abcam, Cambridge, UK); rabbit anti-pSer909-LATS1 (1:100, \#9157, Cell Signaling Technology, Danvers, MA, USA); rabbit anti-pThr210-PLK1 (1:5000, ab155095, Abcam, Cambridge, UK); rabbit anti-YAP (1:20, sc-15407, Santa Cruz Biotechnology, Dallas, TX, USA); mouse anti-RIP1 (1:200, \#610459, BD bioscience, CA, USA); rabbit antiRIP3 (1:250, ab56164, Abcam, Cambridge, UK); rabbit anti-pSer166-RIP1 (1:400, \#44590, Cell Signaling Technology, Danvers, MA, USA); rabbit anti-pSer232-RIP3 (1:100, ab195117, Abcam, Cambridge, UK); rabbit anti-pSer345-MLKL (1:2000, ab196436, Abcam, Cambridge, UK);.Secondary antibodies were as follows: donkey anti-mouse IgG Alexa488 (1:1000, A-21202, Molecular Probes, Eugene, OR, USA), and donkey anti-rabbit IgG Alexa568 (1:1000, A-10042, Molecular Probes, Eugene, OR, USA). Nuclei were stained with DAPI $(0.2 \mu \mathrm{g} / \mathrm{mL}$ in PBS, D523, Dojindo Laboratories, Kumamoto, Japan). For multi-labeling, antibodies were labeled by Zenon Secondary Detection-Based Antibody Labeling Kits as follows: anti-calnexin, anti-RIP3, anti-pSer166-RIP1 and anti-pSer232-RIP3 (Zenon ${ }^{\mathrm{me}}$ Alexa Fluor ${ }^{\mathrm{rm}}$ 555 Rabbit IgG Labeling Kit, Z-25305, Thermo Fisher Scientific, Waltham, MA, USA); anti-MAP2 (Zenon ${ }^{\text {ntw }}$ Alexa Fluor ${ }^{\text {met }} 647$ Rabbit IgG Labeling Kit, Z-25308 and Zenon $^{\text {me }}$ Alexa Fluor ${ }^{\mathrm{m}} 488$ Rabbit IgG Labeling Kit, Z-25302, Thermo Fisher Scientific, Waltham, MA, USA); anti-pSer345-MLKL (Zenon ${ }^{\text {mat }}$ Alexa Fluor ${ }^{\mathrm{rm}} 647$ Rabbit IgG Labeling Kit, Z25308 Thermo Fisher Scientific, Waltham, MA, USA); anti-amyloid $\beta$ (Zenon $^{\mathrm{mm}}$ Alexa Fluor ${ }^{\mathrm{ma}} 488$ Mouse IgG $_{1}$ Labeling Kit, Z-25002, Thermo Fisher Scientific, Waltham, MA, USA). All images were acquired by fluorescence microscopy (Olympus IX70, Olynpus, Tokyo, Japan) or confocal microscopy (FV1200IXGP44, Olympus, Tokyo, Japan).

ELISA evaluation of $\mathbf{A} \boldsymbol{\beta}$ levels in mouse brains. We performed sandwich ELISA using Human $\beta$ Amyloid (1-42) ELISA Kit or Human $\beta$ Amyloid (1-40) ELISA Kit (298-62401 and 292-62301, FUJIFILM Wako PureChemical Corp., Osaka, Japan). Total proteins extracted from $20 \mathrm{mg}$ of mouse cortex tissues by $1 \mathrm{~mL}$ of RIPA buffer (10 mM Tris-HCl pH7.5, $150 \mathrm{mM} \mathrm{NaCl}, 1 \mathrm{mM}$ EDTA, $1 \%$ Triton X-100,
$0.1 \%$ SDS, $0.1 \%$ sodium deoxycholate) were ultra-centrifuged at $100,000 \times g$ at $4{ }^{\circ} \mathrm{C}$ for 1 hour. The supernatants were diluted to $100 \mu \mathrm{L}$ and applied to $\mathrm{A} \beta 1-42$ (extract from $100 \mu \mathrm{g}$ cortex/well) and $\mathrm{A} \beta 1-40$ (extract from $500 \mu \mathrm{g}$ cortex/well) ELISA plates and measured following the manufacturer's instructions.

The ELISA plates were incubated at $4{ }^{\circ} \mathrm{C}$ overnight, washed five times with $1 \mathrm{x}$ wash solution, added with $100 \mu \mathrm{L}$ of HRP-conjugated antibody solution, and incubated at $4{ }^{\circ} \mathrm{C}$ for 1 or $2 \mathrm{~h}$ in A $\beta 1-42$ or A $\beta 1-40$ ELISA kits, respectively. After washed five times by $1 \mathrm{x}$ wash solution, the ELISA plates were added with $100 \mu \mathrm{L}$ of TMB solution, incubated at room temperature for $30 \mathrm{~min}$, and added with $100 \mu \mathrm{L}$ of stop solution. Absorbance was measured at $450 \mathrm{~nm}$ using a plate reader (SPARK $10 \mathrm{M}$, TECAN, Grodig, Austria).

Immuno-electron microscopy. The tissues were fixed with $4 \%$ paraformaldehyde for $12 \mathrm{~h}$, followed by the cryo-protective treatment with $30 \%$ sucrose. The frozen tissue blocks in the cryo-compound were sliced with $20 \mu \mathrm{m}$ thickness with cryostat. Sections were incubated with the $5 \%$ Block Ace (UKB80, DS Pharma Biomedical, Osaka, Japan) solution with $0.01 \%$ Saponin in $0.1 \mathrm{M}$ PB for an hour, and stained with primary rabbit anti-pSer46-MARCKS (1:1000, ordered from GL Biochem Ltd., Shanghai, China) for $72 \mathrm{~h}$ at $4{ }^{\circ} \mathrm{C}$, followed by the incubation with nanogold conjugated goat anti-rabbit secondary antibody (1:100, N-24916, Thermo Fisher Scientific, Waltham, MA, USA) for $24 \mathrm{~h}$ at $4{ }^{\circ} \mathrm{C}$. After $2.5 \%$ glutaraldehyde fixation in PB, nanogold signals were enhanced with R-Gent SE-EM Silver Enhancement Reagents (500.033, Aurion, Wageningen, Netherlands) for $40 \mathrm{~min}$ at $25^{\circ} \mathrm{C}$. Stained sections were post-fixed with $1.0 \% \mathrm{OsO} 4$ for $90 \mathrm{~min}$ at $25^{\circ} \mathrm{C}$, dehydrated through graded series of ethanol and embedded in Epon. Ultrathin sections $(70 \mathrm{~nm})$ were prepared with ultramicrotome (UC7, Leica, Wetzlar, Germany) and stained with uranyl acetate and lead citrate. The sections were observed under a transmission EM (JEOL model 1400 plus, JEOL Ltd., Tokyo, Japan).

Immunoprecipitation. Mouse cerebral cortex was lysed in a homogenizer with RIPA buffer (10 mM Tris-HCl, pH 7.5, $150 \mathrm{mM} \mathrm{NaCl}, 1 \mathrm{mM}$ EDTA, $1 \%$ Triton X100, $0.1 \%$ SDS, $0.1 \%$ DOC, $0.5 \%$ protease inhibitor cocktail (539134, Calbiochem, San Diego, CA, USA)). Lysates were rotated for $60 \mathrm{~min}$ at $4^{\circ} \mathrm{C}$, and then centrifuged $\left(12,000 \mathrm{~g} \times 1 \mathrm{~min}\right.$ at $\left.4{ }^{\circ} \mathrm{C}\right)$. Supernatant $(250 \mu \mathrm{g})$ was incubated with a $50 \%$ slurry of protein G-Sepharose beads $(100 \mu \mathrm{L}, 17061801$, GE Healthcare,, Chicago, IL, USA), followed by centrifugation $\left(2000 \mathrm{~g} \times 3 \mathrm{~min}\right.$ at $\left.4^{\circ} \mathrm{C}\right)$. Supernatants were incubated with $1 \mathrm{\mu g}$ of antibody for $16 \mathrm{~h}$ at $4{ }^{\circ} \mathrm{C}$ with rotation. Antibodies were as follows: rabbit anti-YAP (1:100, \#14074, Cell Signaling Technology, Danvers, MA, USA); mouse anti-amyloid $\beta$ (1:5000, clone 82E1, \#10323, IBL, Gunma, Japan). Protein G-Sepharose was added to samples and rotated for $4 \mathrm{~h}$ at $4{ }^{\circ} \mathrm{C}$, and then the beads were washed three times with RIPA buffer. Equal volume of sample buffer (125 mM Tris-HCl, pH 6.8, 4\% SDS, 10\% glycerol, 0.005\% BPB, 5\% 2mercaptoethanol) was added, and the samples were boiled at $100^{\circ} \mathrm{C}$ for $10 \mathrm{~min}$ before SDS-PAGE.

Dot blot. Protein concentrations in samples were measured using the BCA Protein Assay Regent (Micro BCA Protein Assay Reagent kit, 23225, Thermo Fisher Scientific, Waltham, MA, USA). After the membranes (Immobilon-P, IPVH00010, Merck Millipore, Burlington, MA, USA) were washed by TBS ( $20 \mathrm{mM}$ Tris-HCl/ $\mathrm{pH} 7.5,500 \mathrm{mM} \mathrm{NaCl}$ ), samples of $2.5 \mu \mathrm{g} / 25 \mu \mathrm{L}$ were dropped on membranes using Bio-Dot Apparatus (1706545, Bio-Rad Laboratories Laboratories, CA, USA) and left to stand overnight. Next, the membranes were blocked with 5\% skim milk in TBST (10 mM Tris/HCl pH 8.0, $150 \mathrm{mM} \mathrm{NaCl}, 0.05 \%$ Tween-20), and reacted with the following primary and secondary antibodies diluted in Can Get Signal solution (NKB-101, Toyobo, Osaka, Japan). ECL prime (RPN2232, GE Healthcare, Chicago, IL, USA) or ECL select (RPN2235, GE Healthcare, Chicago, IL, USA) were used to detect the bands using LAS500 (29005063, GE Healthcare, Chicago, IL, USA). Primary and secondary antibodies were diluted as follows: mouse anti-amyloid $\beta$, (1:5000, clone 82E1, \#10323, IBL, Gunma, Japan); HRP-conjugated anti-mouse IgG (1:5000, NA931VA, GE Healthcare, Chicago, IL, USA). 
a
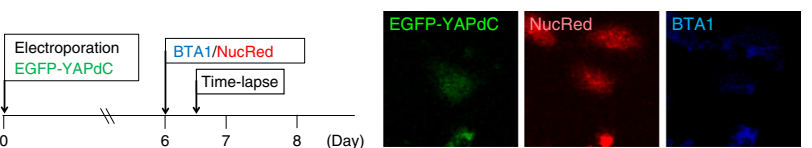

b
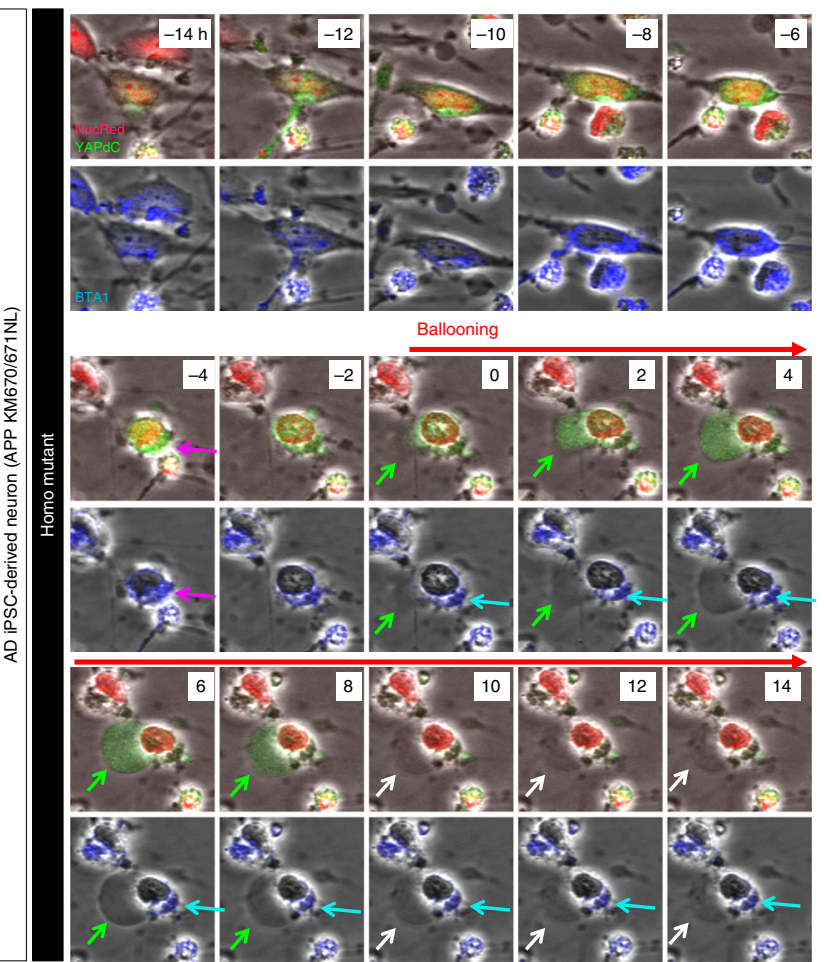

Ballooning
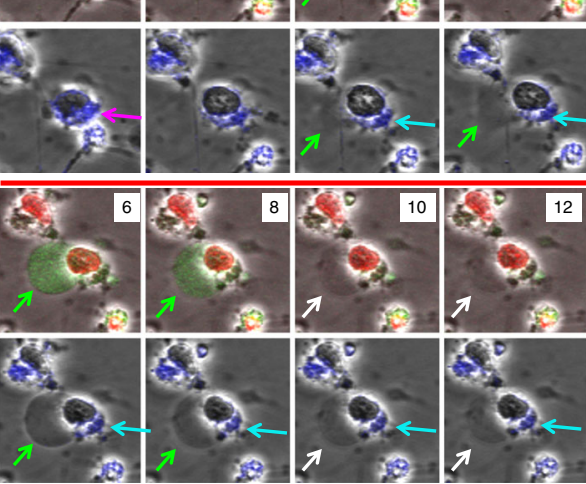

C

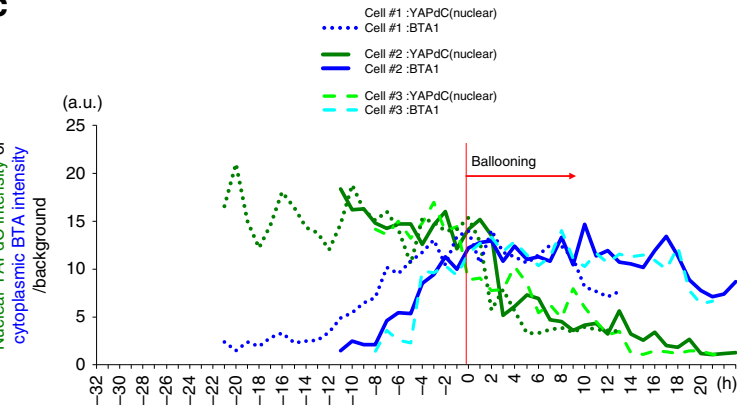

d

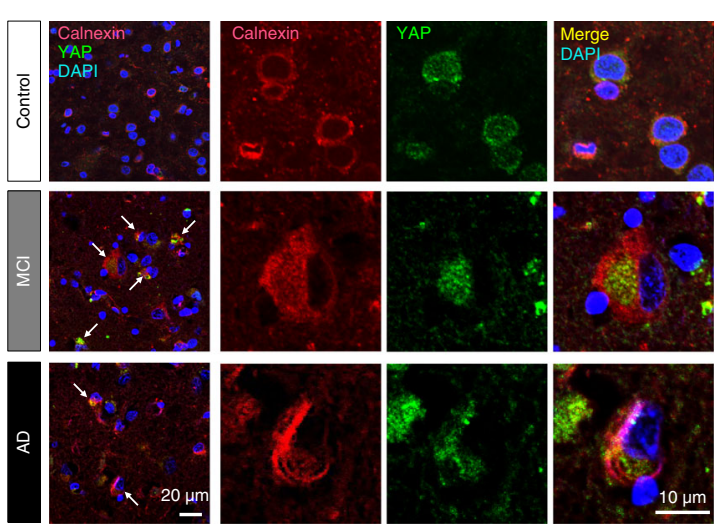

Western blot. Protein concentration of samples was measured using the BCA Protein Assay Regent (Micro BCA Protein Assay Reagent kit, 23225 Thermo Fisher Scientific, Waltham, MA, USA). After samples were separated by SDS-PAGE, they were transferred onto polyvinylidene difluoride membranes (Immobilon-P, IPVH00010, Merck Millipore, Burlington, MA, USA) using the semi-dry method. Next, the membranes were blocked with $5 \%$ skim milk in TBST (10 mM Tris/ $\mathrm{HCl} \mathrm{pH} \mathrm{8.0,150} \mathrm{mM} \mathrm{NaCl,} \mathrm{0.05 \%} \mathrm{Tween-20),} \mathrm{and} \mathrm{reacted} \mathrm{with} \mathrm{the}$
Fig. 8 Time lapse imaging of a single neuron elucidates pathological cascade. a Time lapse imaging protocol of a single neuron to analyze relationship between intracellular localization of YAP, intracellular $A \beta$ and ER ballooning. Representative images of the same cells (similar to the cells in b) are shown in right panels. b Time lapse imaging of an iPSC-derived neurons carrying APP mutations (homozygous mutants carrying APP KM670/671NL). Nuclear YAP was shifted to intracellular A $\beta$ in the cytoplasm (magenta arrow) and further to ballooned ER (green arrow). YAP was released to extracellular space via leakage of ballooned ER (white arrow) while intracellular $A \beta$ remains as aggregates (blue arrow). The details were described in the text. c Chronological change of nuclear YAPdeltaC intensity and cytoplasmic BTA intensity in three iPSC-derived neurons carrying APP mutations. d Immunohistochemistry of human cerebral cortex with anti-calnexin, an ER membrane marker, and anti-YAP antibodies. Abnormal localizations of YAP in the cytoplasm or ballooned ER were observed frequently observed in $\mathrm{MCl}$ patients and at a low frequency in AD patients (white arrow), consistently with the findings in iPSC-derived neurons carrying APP mutations. Source data are provided as a "Source Data file".

following primary and secondary antibodies diluted in Can Get Signal solution (NKB-101, Toyobo, Osaka, Japan). Bands were visualized using ECL prime (RPN2232, GE Healthcare, Chicago, IL, USA) or ECL select (RPN2235, GE Healthcare, Chicago, IL, USA). Primary and secondary antibodies were diluted as follows: rabbit-anti-YAP (H-125) (1:3000, sc-15407, Santa Cruz Biotechnology, Dallas, TX, USA); $\beta$-actin(C-4) (1:3000, sc-47778, Santa Cruz Biotechnology, Dallas, TX, USA); mouse anti-amyloid $\beta,(1: 1000$, clone 82E1, \#10323, IBL Gunma, Japan); rabbit-anti-YAPdeltaC $(1: 9000)^{17}$; mouse anti-RIP1 (1:1000, 610459, BD bioscience, CA, USA); rabbit anti-RIP3 (1:1000, ab56164, Abcam, Cambridge, UK); anti-pSer166-RIP1 (1:1000, \#44590, Cell Signaling Technology, Danvers, MA, USA); anti-pSer232-RIP3 (1:1000, ab195117, abcam, Cambridge, UK); anti-pSer46-MARCKS antibody (1:100000, ordered from GL Biochem Ltd., Shanghai, China); anti-histone H3 antibody (1:1000, 630767, Merck, Darmstadt, Germany) HRP-conjugated anti-mouse IgG (1:3000, NA931VA, GE Healthcare, Chicago, IL, USA); and HRP-conjugated anti-rabbit IgG (1:3000, NA934VS, GE Healthcare, Chicago, IL, USA).

Immunocytochemistry. iPS-derived neurons were fixed in 4\% PFA, and then permeabilized by incubation with $0.1 \%$ Triton X-100 in PBS for $10 \mathrm{~min}$ at room temperature (RT). After blocking with blocking buffer $(50 \mathrm{mM}$ Tris- $\mathrm{HCl} \mathrm{pH} 6.8$, $150 \mathrm{mM} \mathrm{NaCl}$, and $0.1 \%$ Triton X-100) containing $5 \mathrm{mg} / \mathrm{mL}$ BSA for $60 \mathrm{~min}$ at RT, sections were incubated with primary antibody for $60 \mathrm{~min}$ or $180 \mathrm{~min}$ (only for $6 \mathrm{E} 10$ ), and finally with secondary antibodies for $60 \mathrm{~min}$ at RT. The antibodies used for immunocytochemistry were diluted as follows: rabbit-anti-YAP $(1: 100, \# 14074$ S, Cell Signaling Technology, Danvers, MA, USA), which was raised against amino acids around Pro435 of human YAP isoform 1; rabbit-anti-YAP (1:200, sc-15407, Santa Cruz Biotechnology, Dallas, TX, USA), which was raised against amino acids 206-330 of human YAP; rabbit-anti-YAPdeltaC $(1: 2000)^{17}$; mouse-anti-A $\beta$ (1:250, clone 6E10, SIG-39300, Covance, NJ, USA); anti-pSer46-MARCKS antibody (1:2000, ordered from GL Biochem Ltd., Shanghai, China); Cy3-conjugated antimouse IgG (1:500, 715-165-150, Jackson Laboratory, Bar Harbor, ME, USA); and Alexa Fluor 488-conjugated anti-rabbit IgG (1:1000, A11008, Molecular Probes, Eugene, OR, USA).

TEAD-YAP transcriptional activity. Neurospheres differentiated from human iPS cells (with or without APP mutations) ${ }^{37}$ were dissociated in TrypLE ${ }^{\text {Tx }}$ Select (12563-011, Thermo Fisher Scientific, Waltham, MA, USA) containing $10 \mu \mathrm{M}$ Y27632 (253-00513, Wako, Osaka, Japan). In total $4 \times 10^{5}$ cells, centrifuged, and suspended in $20 \mu \mathrm{L}$ nucleofector solution (P3 Primary Cell $4 \mathrm{D}-\mathrm{Nucleofector}^{\mathrm{Th}} \mathrm{X}$ Kit V4XP-3012, LONZA, NJ, USA). In total $1 \mu \mathrm{g}$ of pLL3.7-ires-GFP-TEAD-responsive-H2B-mCherry plasmid ${ }^{38}$ (generous gift from Prof. Yutaka Hata, Tokyo Medical and Dental University) was added to the cell suspension and electroporated into cells by 4D-Nucleofector (pulse program: CV-110) (4D-Nucleofector Core Unit, \#AAF-1002B, LONZA, NJ, USA). The electroporated cells were cultured on Lab-Tek II chambered coverglass coated with poly-L-ornithine (P3655, Sigma-Aldrich, St. Louis, MO, USA) and laminin (23016015, Thermo Fisher Scientific, Waltham, MA, USA) in DMEM/F12 (D6421, Sigma-Aldrich, St. Louis, MO, USA) supplemented with B27 (17504044, Thermo Fisher Scientific, Waltham, MA, USA), Glutamax (35050061, Thermo Fisher Scientific, Waltham, MA, USA), and penicillin/streptomycin (15140-122, Thermo Fisher Scientific, Waltham, MA, USA). After differentiation to neurons for seven days, time-lapse images of pTEAD-driven mCherry and pCMV-driven EGFP were acquired by Olympus FV10i-W (Olympus, Tokyo, Japan) at $30 \mathrm{~min}$ intervals for $36 \mathrm{~h}$. Cells were co- 
a

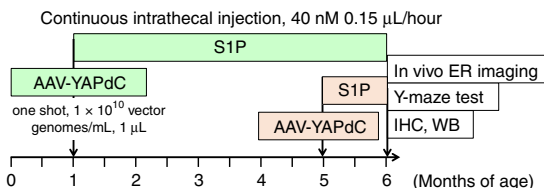

b
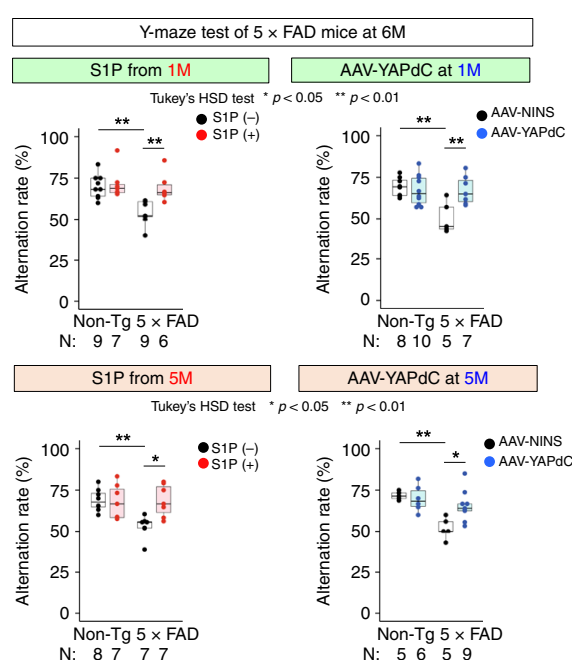

C

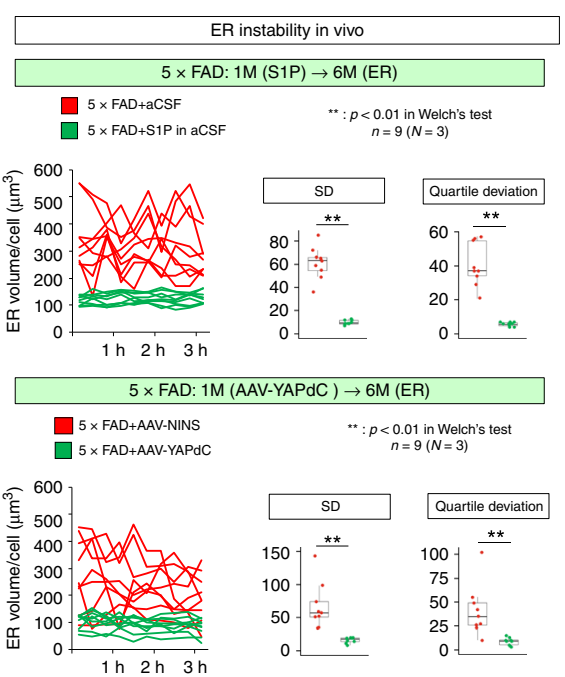

d
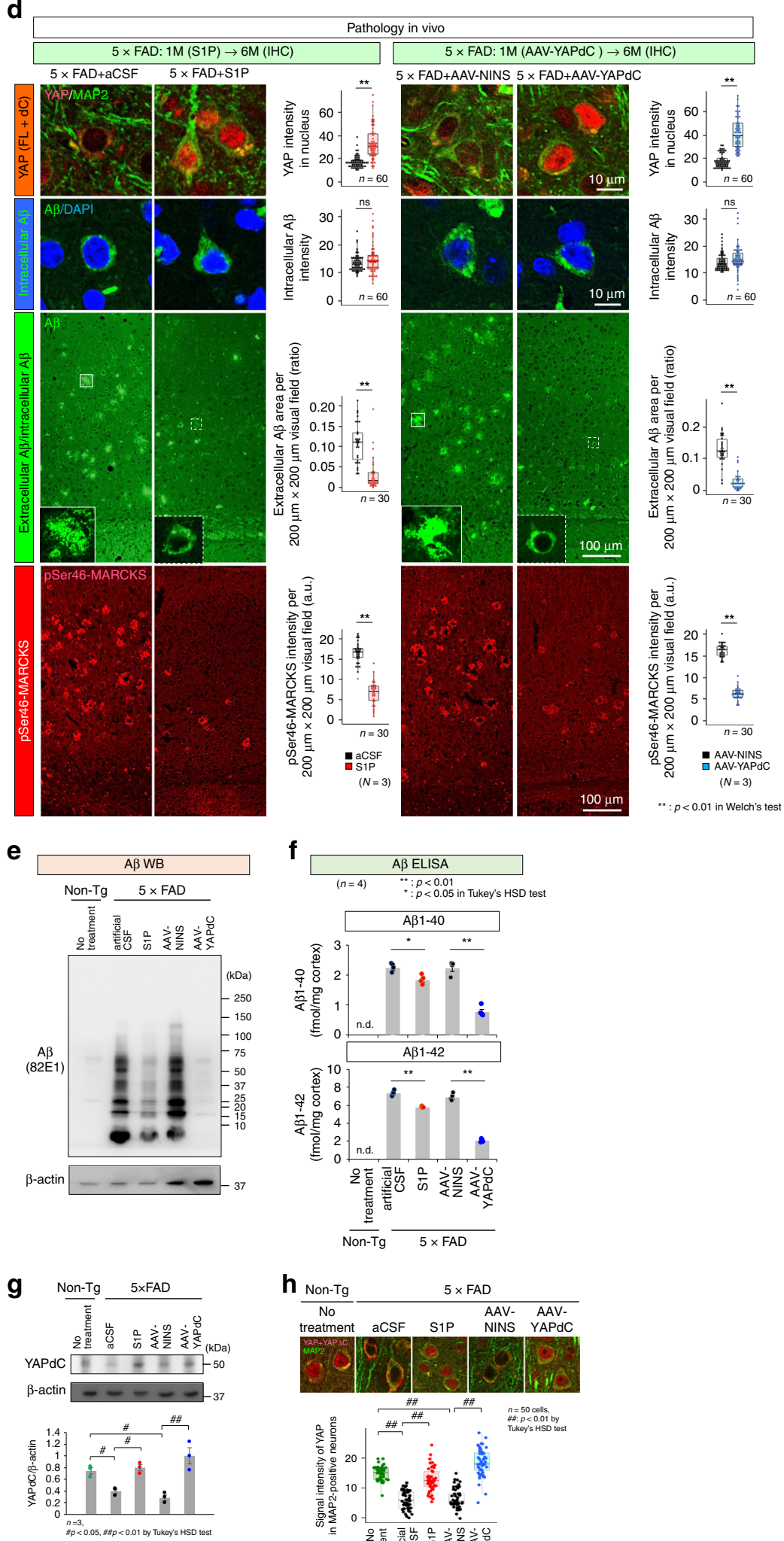

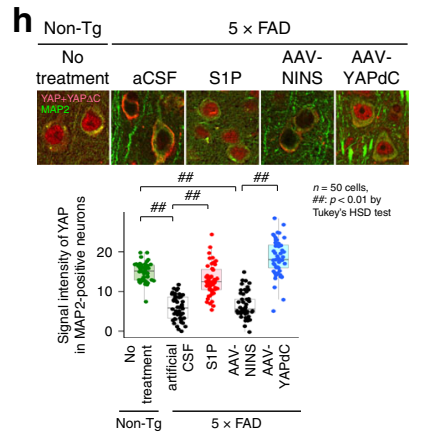

stained with ER-Tracker" Blue-White DPX (\#E12537, Thermo Fisher Scientific, MA, USA) and ER signals were obtained in parallel. Measurement of signal intensity was performed using Fluoview (Olympus, Tokyo, Japan) software. The chamber was kept at $37^{\circ} \mathrm{C}$ with $5 \% \mathrm{CO}_{2}$ during the experiment.
siRNA-mediated knockdown of YAP in iPS-derived neurons in vitro. In total 5 pmol (67.6 ng) of human YAP-siRNA (sc-38637, Santa Cruz Biotechnology, Dallas, TX, USA) or 5 pmol of Trilencer-27 universal scrambled negative control siRNA duplex (\#SR30004, OriGene, Rockville, MD, USA) was transfected into human 
Fig. 9 S1P and YAPdeltaC rescue ER instability and cognitive impairment in AD model mice. a Experimental protocol for the rescue effect of S1P or YAPdeltaC (YAPdC) on ER instability and cell death in 5xFAD mice. Two protocols, administration before symptomatic onset (1 month) or administration just after onset (5 months), were used. AAV-NINS: AAV-CMV-no insert. b Alteration rate in Y-maze test of 6-month-old 5xFAD mice that had been treated with S1P from 1 month (upper left panel) or 5 months (lower left panel) or injected with AAV-YAPdeltaC at 1 month (upper right panel) or 5 months (lower right panel). P-values were determined by Tukey's HSD test, ${ }^{\star} p<0.05,{ }^{\star \star} p<0.01$. N: shown below graphs. c ER instability was rescued at 6 months following treatment from 1 month with S1P or YAPdeltaC. P-values were determined by Welch's test, ${ }^{\star *} p<0.01$ ( $N=3$ mice, $n=9$ cells). d Pathological examination at 6 months following treatment from 1 month with S1P or YAPdeltaC. Staining of YAP with the SC-15407 antibody detecting YAP-FL and YAPdeltaC, intracellular/extracellular A $\beta$, and pSer46MARCKS is shown. Right graphs show quantification of the four stains before and after the treatment. P-values were determined by Welch's test, ${ }^{\star \star} p<0.01(N=3$ mice, $n=30$ or 60$)$. e Western blot with anti-A $\beta$ antibody (82E1) confirmed the effect of S1P and AAV-YAPdC on A $\beta$ burden in 5xFAD mice. $\mathbf{f}$ ELISA of A $1-40$ and A $\beta 1-42$ consistently showed the effect of S1P and AAV-YAPdC reducing $A \beta$ burden in 5xFAD mice. $P$-values were determined by Tukey's HSD test, ${ }^{\star} p<0.05,{ }^{\star \star} p<0.01(n=4$ mice). $\mathbf{g}$ Western blot confirming the increase of YAPdeltaC protein in cerebral cortex tissue of 5xFAD mice after AAV-YAPdeltaC infection. S1P also increased YAPdeltaC. P-values were determined by Tukey's HSD test, $\# p<0.05, \# \# p<0.01$ ( $n=3$ tests). $\mathbf{h}$ Immunohistochemistry of cerebral cortex tissue of $5 x F A D$ mice supported the increase of total YAP after S1P and AAV-YAPdeltaC treatments. P-values were determined by Tukey's HSD test, \#\#p $<0.01$ ( $n=50$ cells). Box plots show the median, quartiles and whiskers that represent 1.5 5 the interquartile range. Bar graphs indicate average and mean S.E.M., together with the corresponding data points. Source data are provided as a "Source Data file".

neurons differentiated from normal iPS cells (ASE-9203, Applied StemCell, Milpitas, CA, USA) using Viromer ${ }^{\circledR}$ BLUE (TT100300, OriGene, Rockville, MD, USA). Before transfection, siRNA was labeled with Label IT ${ }^{\oplus}$ siRNA Tracker ${ }^{\text {ix }}$ Fluorescein Kit without Transfection Reagent (MIR7216, Mirus, WI, USA) according to the manufacturer's procedures. Eighteen hours later, cells were stained with ER-Tracker" Red (BODIPY ${ }^{\mathrm{mm}}$ TR Glibenclamide) (E34250, Thermo Fisher Scientific, MA, USA) and NucRed ${ }^{\text {tex }}$ Live 647 ReadyProbes $^{\mathrm{Tw}}$ Reagent (R37106, Thermo Fisher Scientific, MA, USA) for $60 \mathrm{~min}$ at $37^{\circ} \mathrm{C}$. Time-lapse images of iPSderived neurons were acquired at $\times 60$ magnification on an Olympus FV10i-W (Olympus, Tokyo, Japan) at $15 \mathrm{~min}$ intervals for $24 \mathrm{~h}$. The chamber was kept at $37^{\circ} \mathrm{C}$ with $5 \% \mathrm{CO}_{2}$. The ratio of cell death patterns was counted cells transfected labeled-siRNA. To validate knockdown efficiency of siRNA, cells were fixed and collected. Each sample was used for Immunocytochemistry and western blot.

siRNA-mediated knockdown of YAP in mice in vivo. Before siRNA injection, siRNA was labeled with Label IT ${ }^{\circledast}$ siRNA Tracker ${ }^{\mathrm{rm}}$ Fluorescein Kit without Transfection Reagent (MIR7216, Mirus, WI, USA) according to the manufactural procedures. Under anesthesia with $1 \%$ isoflurane, we injected $300 \mathrm{ng}$ Fluoresceinlabeled-siRNA (mouse YAP-siRNA, sc-38638, Santa Cruz Biotechnology, Dallas, TX, USA) or Trilencer-27 universal scrambled negative control siRNA duplex (sc38637, Santa Cruz Biotechnology, Dallas, TX, USA) into retrosplenial cortex (anteroposterior, $-2.0 \mathrm{~mm}$ form bregma; lateral, $0.6 \mathrm{~mm}$; depth, $1 \mathrm{~mm}$ ) at a volume of $1 \mu \mathrm{l}$ using in vivo jetPEI (201-10 G, Polyplus-transfection, Illkirch, France) at 5 months of age (21 weeks). After $16 \mathrm{~h}$, live-cell imaging of ER was performed by two-photon microscopy followed by the method listed above ("In vivo imaging of neuronal $\mathrm{ER}$ and $\mathrm{A} \beta$ ").

After dissection, mouse cerebral cortexes were fixed in 4\% PFA for overnight at $4{ }^{\circ} \mathrm{C}$. Tissue sections were prepared at $30 \mu \mathrm{m}$ thickness using microtome (MICROM HM650V, Thermo Fisher Scientific, Waltham, MA, USA), and stained in floating condition. In brief, sections were incubated with blocking solution $(10 \%$ FBS and $0.3 \%$ triton- $\mathrm{X}$ in PBS) for $30 \mathrm{~min}$ at RT, and with a primary antibody (antiYAP antibody (1:100, sc-15407, Santa Cruz Biotechnology, Dallas, TX, USA); pSer46-MARCKS (1:2000, ordered from GL Biochem Ltd., Shanghai, China)) overnight at $4{ }^{\circ} \mathrm{C}$. After three times of washing with PBS for $5 \mathrm{~min}$, the sections were incubated with goat-anti rabbit IgG Alexa568 for $1 \mathrm{~h}$ at room temperature, and with DAPI for $5 \mathrm{~min}$.

Pathological cascade analysis of a single cell neuron. pEGFP-YAPdeltaC was generated by subcloning EcoRI-SalI fragment digested from pBS-YAPdeltaC ${ }^{17}$ into pEGFP-C1 (\#6084-1, Clontech, Mountain View, CA, USA). Neurospheres differentiated from human iPS cells (with or without APP mutations) ${ }^{37}$ were dissociated in TrypLE ${ }^{\mathrm{m}}$ Select (12563-011, Thermo Fisher Scientific, Waltham, MA, USA) containing $10 \mu \mathrm{M}$ Y27632. $4 \times 10^{5}$ cells, centrifuged, and resuspended in $20 \mu \mathrm{L}$ nucleofector solution (P3 Primary Cell 4D-Nucleofector ${ }^{\mathrm{rt}} \mathrm{X}$ Kit, V4XP-3012, LONZA, NJ, USA). One $\mu \mathrm{g}$ of pEGFP-YAPdeltaC was added to the cell suspension and electroporated into cells by $4 \mathrm{D}-\mathrm{Nucleofector}$ (pulse program: CV-110) $(4 \mathrm{D}-$ Nucleofector Core Unit, AAF-1002B, LONZA, fNJ, USA). The electroporated cells were cultured on Lab-Tek II chambered coverglass coated with poly-L-ornithine (P3655, Sigma-Aldrich, St. Louis, MO, USA) and laminin (23016015, Thermo Fisher Scientific, Waltham, MA, USA) in DMEM/F12 (D6421, Sigma-Aldrich, St. Louis, MO, USA) supplemented with B27 (17504044, Thermo Fisher Scientific, Waltham, MA, USA), Glutamax (35050061, Thermo Fisher Scientific, Waltham, MA, USA), and penicillin/streptomycin (15140-122, Thermo Fisher Scientific, Waltham, MA, USA). After differentiation to neurons for 6 days, time-lapse images of EGFP-YAPdeltaC were acquired by Olympus FV10i-W (Olympus, Tokyo, Japan) at $30 \mathrm{~min}$ intervals for $36 \mathrm{~h}$. Cells were co-stained with NucRed ${ }^{\mathrm{mat}}$ Live 647 ReadyProbes $^{\mathrm{sw}}$ Reagent (R37106, Thermo Fisher Scientific, MA, USA) and $100 \mathrm{nM}$ 2-(4'-methylaminophenyl) benzothiazole (BTA1, B9934, Sigma-Aldrich, MO, USA). The chamber was kept at $37^{\circ} \mathrm{C}$ with $5 \% \mathrm{CO}_{2}$ during the experiment. Measurement of signal intensity was performed using the ImageJ software (Ver. $1.45 \mathrm{~s})$.

Generation of AAV-YAPdeltaC. The AAV vector plasmid carries an expression cassette consisting of a human CMV promoter, the first intron of human growth hormone 1, cDNA encoding rat YAPdeltaC (ins61; accession no. DQ186898.2), woodchuck hepatitis virus post-transcriptional regulatory element (WPRE), and a simian virus 40 polyadenylation signal sequence (SV40 poly[A]) between the inverted terminal repeats of the AAV1 genome. To generate the vectors, AAV2 rep and AAV1 vp expression plasmids, and an adenoviral helper plasmid (pHelper, 240071-54, Agilent Technologies, CA, USA) were co-transfected into HEK293 cells by the calcium phosphate co-precipitation method. The recombinant viruses were purified by isolation from two sequential continuous $\mathrm{CsCl}$ gradients. Viral titers were determined by $\mathrm{qPCR}$.

Intrathecal injection of AAV and S1P. In pharmacological rescue experiments, sphingosine-1-phosphate (S1P, $40 \mathrm{nM}$ Sigma-Aldrich, St. Louis, MO, USA) or artificial cerebrospinal fluid (3525, aCSF, R\&D systems, MN, USA) was administered to mice at 4 weeks old into the subarachnoid space via osmotic pump $(0.15 \mu \mathrm{L} / \mathrm{h}$, model 2006, ALZET, Cupertino, Canada) for 28 days. For the YAP rescue experiment, AAV1-YAPdeltaC or AAV-CMV-NINS (titer: $1 \times 10^{10}$ vector genomes $/ \mathrm{mL}, 1 \mu \mathrm{L}$ ) was injected into retrosplenialdysgranular cortex at $-2.0 \mathrm{~mm}$ from bregma, mediolateral $0.6 \mathrm{~mm}$, depth $1 \mathrm{~mm}$. Two days after drug injection, imaging was performed 10 times at 20 min intervals using the following method.

In vivo imaging of neuronal ER and $\mathbf{A} \boldsymbol{\beta}$. The skull was thinned with a high-speed micro-drill at the surface of the mouse splenial cortex ${ }^{57}$. The head of each mouse was immobilized by attaching the head plate to a custom machine stage mounted on the microscope table. Two-photon imaging was performed using FV1000MPE2 (Olympus, Tokyo, Japan) equipped with an upright microscope (BX61WI, Olympus, Tokyo, Japan), a water-immersion objective lens (XLPlanN25xW; numerical aperture, 1.05, Olympus, Tokyo, Japan), and a pulsed laser (MaiTaiHP DeepSee, Spectra Physics, Santa Clara, CA, USA). Four hours before imaging, BTA1 (100 nM, B-9934, Sigma-Aldrich, MO, USA) and ER-Tracker ${ }^{\mathrm{II}}$ Red (100 nM, E34250, Thermo Fisher Scientific, MA, USA) were injected in a volume of $1 \mu \mathrm{L}$ into RSD at $-2.0 \mathrm{~mm}$ from bregma, mediolateral $\pm 0.6 \mathrm{~mm}$, depth $1 \mathrm{~mm}$, under anesthesia with $1 \%$ isoflurane. Both BTA1 and ER were excited at $750 \mathrm{~nm}$ and scanned at $495-540 \mathrm{~nm}$ and $575-630 \mathrm{~nm}$, respectively. High-magnification imaging $(101.28 \times 101.28 \mu \mathrm{m} ; 1024 \times 1024$ pixels; $1 \mu \mathrm{m}$ Z-step; $60-80$ slices along Z-axis $)$ of the cortical layer I in RSD was performed with a $2 \times$ digital zoom through the thinned-skull window in the retrosplenial cortex ${ }^{57}$. Images of BTA1 and ERTracker $^{\mathrm{mm}}$ Red were analyzed according to three parameters: ER and BTA1 signal intensity, ER or BTA1 puncta volume, and number of ER-positive cells or BTA1positive cells per imaging volume. Measurement of ER signal intensity was performed using the ImageJ software (Ver. $1.45 \mathrm{~s}$ ). Total ER puncta volumes belonging to a single neuron were quantified by IMARIS (Bitplane, Zurich, Switzerland).

Neurons derived from genome-edited human iPS cells. Human normal iPS cells (ASE-9203, Applied StemCell, Milpitas, CA, USA) were transfected with a mixture of plasmids expressing gRNA (5'-GGAGATCTCTGAAGTGAAGATGG-3') and the Cas9 gene along with single-stranded oligodeoxynucleotides (for human APP KM670/671NL, 5'-TTGGTTGTCCTGCATACTTTAATTATGATGTAATACAG GTTCTGGGTTGACAAATATCAAGACGGAGGAGATCTCTGAAGTGAATCT GGATGCAGAATTCCGACATGACTCAGGATATGAAGTTCATCATCAAAAA 
a

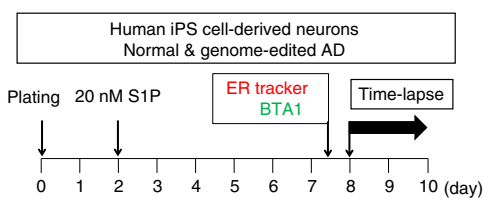

b
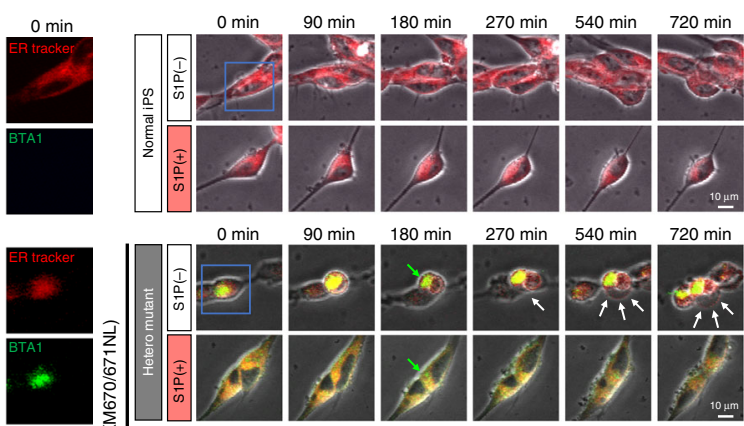

$90 \mathrm{~min}$
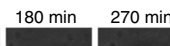

$540 \mathrm{~min}$

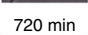

$720 \mathrm{~min}$

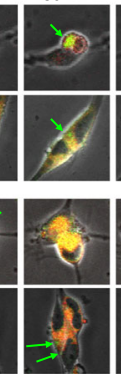

C
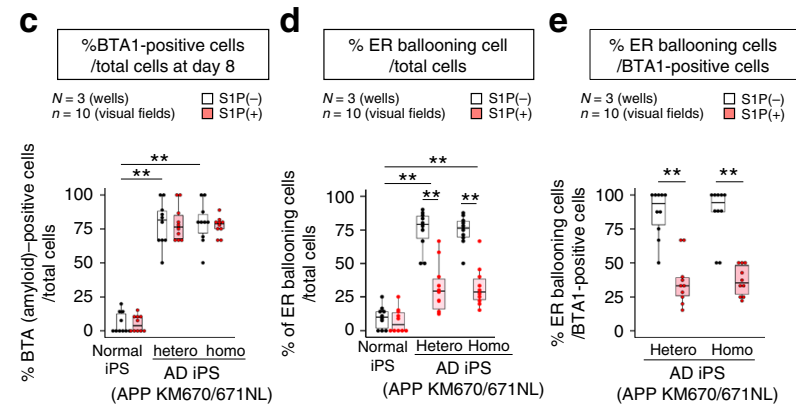

$\mathbf{k}$
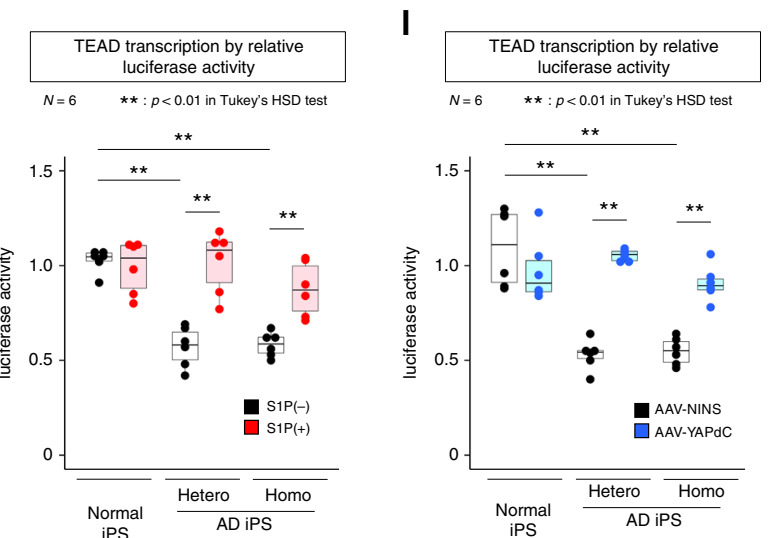

TTGGTACGTAAAATAATTTACCTCTTTC-3') for donor DNA. The Cas9 gene was fused to the 2 A peptide and GFP gene. Cells were electroporated using a Neon system (MPK5000, Thermo Fisher Scientific Inc., MA, USA) with the following conditions: $1200 \mathrm{~V}, 30 \mathrm{~ms}$, one pulse. Cells were selected with $0.4 \mu \mathrm{g} / \mathrm{mL}$ puromycin for $24-48 \mathrm{~h}$ after transfection, and then subjected to a colony cloning process by picking and seeding each visible GFP-positive colony into a well of a 96well plate. The cells were allowed to grow for 7-10 days, or until a conveniently f

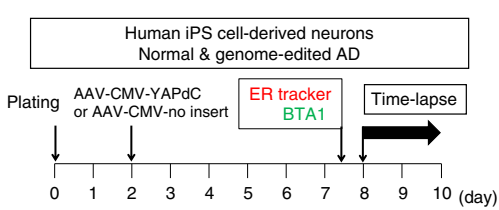

g

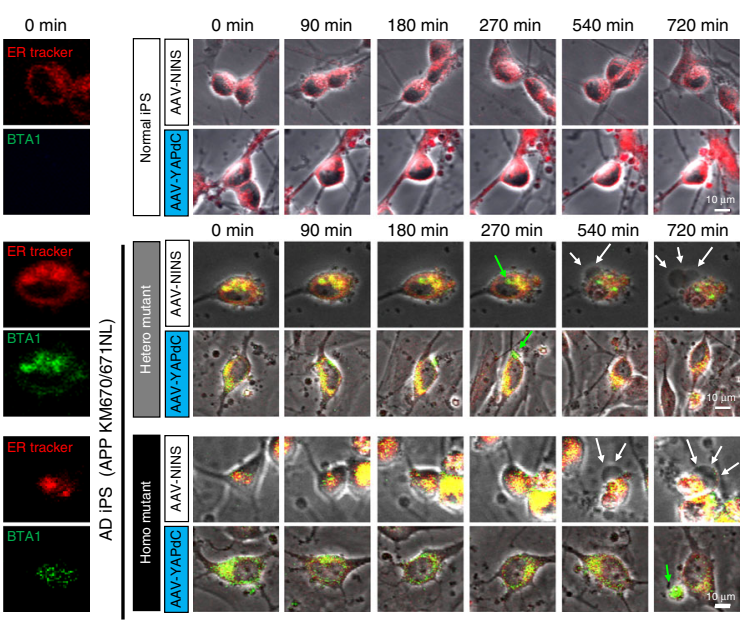

h
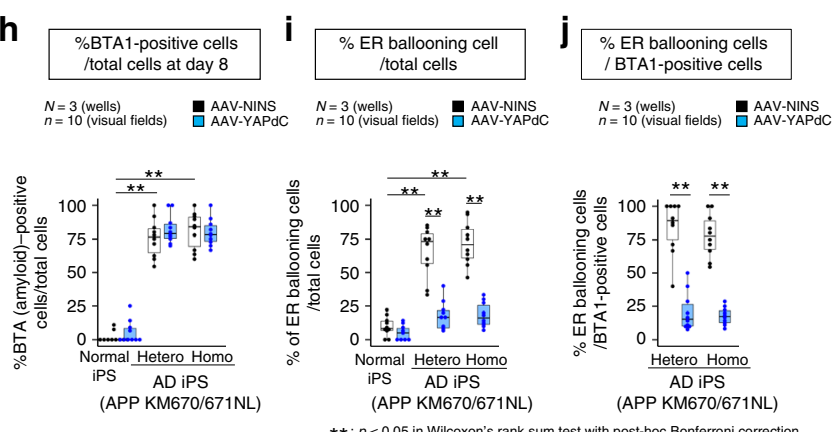

m $\star *: p<0.05$ in Wilcoxon's rank sum test with post-hoc Bonferroni correction

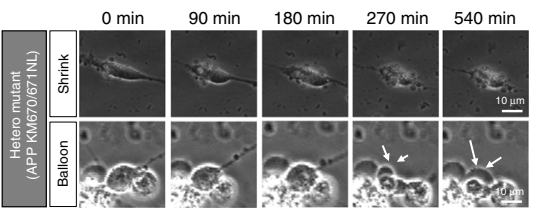

n
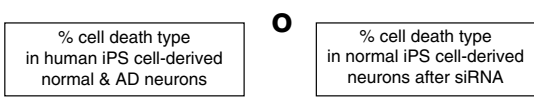

Balloon Rupture Shrink

$\#$ : $p<0.01$ in Tukey's HSD test

$N=3$ (wells)
$n=10$ (visual fields)

$\star \star: ~ p<0.01$ in Wilcoxon's rank sum test

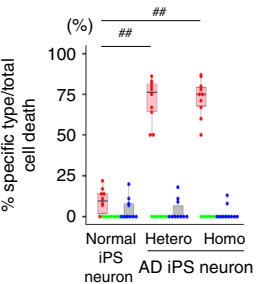

$\begin{aligned} & N=3 \text { (wells) } \\ & n=10 \text { (visual fields) }\end{aligned}$
(a)

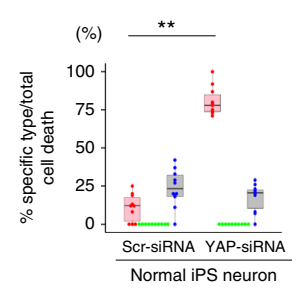

sized colony was formed. A portion of cells from each colony was subjected to genotype analysis. Briefly, genomic DNA from single-cell colonies was isolated and used to amplify a $308 \mathrm{bp}$ DNA fragment using primers 5'-GCATGTATTTAAAG GCAGCAGAAGC- $3^{\prime}$ and $5^{\prime}$-CAATGCTTGCCTATAGGATTACCATGAAAAC ATG-3'. PCR fragments were subjected to Sanger sequencing. Positive clones were expanded, and a portion of cells was resubmitted for sequencing to confirm the desired genotype. Primers are listed in Supplementary Table 3. 
Fig. 10 S1P and YAPdeltaC rescue ER instability and necrosis in genome-edited iPSC-derived AD neurons. a Protocol for the rescue effect of S1P on ER instability and cell death. $\mathbf{b}$ Time-lapse images of non-treated iPSC-derived AD neurons exhibited ER ballooning (white arrows) and rupture. S1P treatment stabilized the ER and decreased necrosis. A $\beta$ accumulation in ER (yellow area) and the leak to cytoplasm (green arrow). Representative images are shown in Supplementary Movie 7-12. c S1P did not change \%BTA1-positive iPSC-derived AD neurons. d, e Suppressive effect of S1P on ER ballooning and necrosis of iPSC-derived AD neurons $\mathbf{d}$ and BTA1-positive neurons e. $\mathbf{f}$ Protocol for the rescue effect of YAPdeltaC on ER instability and cell death. AAV-NINS: AAVCMV-no inset. $\mathbf{g}$ Time-lapse images showed ER ballooning (white arrows) and rupture of iPSC-derived AD neurons, which were suppressed by YAPdeltaC expression. A $\beta$ was mainly accumulated in ER (yellow area), but a portion leaked into the cytoplasm (green arrow). Representative images are shown in Supplementary Movie 13-18. h YAPdeltaC did not change \%BTA1-positive iPSC-derived AD neurons. i Suppressive effect of YAPdeltaC on ER ballooning and necrosis in iPSC-derived AD neurons $\mathbf{i}$ and BTA1-positive neurons $\mathbf{j}$. $\mathbf{k}$, I. Rescue of transcriptional function, as determined using TEAD-responsive element reporter plasmid in iPSC-derived neurons. S1P $\mathbf{k}$ and YAPdeltaC I restored TEAD-YAP/YAPdeltaC-dependent transcription, which was suppressed in iPSC-derived AD neurons. ${ }^{\star \star} p<0.01$ ( $N=6$ wells), Tukey's HSD test. $\mathbf{m}$ Time-lapse images of shrinkage apoptosis and ballooning necrosis (arrow). $\mathbf{n}$ The ratio of each type of cell death (shrinkage apoptosis, rupture necrosis and ballooning necrosis) to total neurons that occurred naturally during $24 \mathrm{~h}$ of time-lapse imaging in normal and iPSC-derived AD neurons. ${ }^{\#} p<0.01$ ( $N=3$ wells, $n=10$ visual fields), Tukey's HSD test. $\mathbf{0}$. The ratio of each type of cell death to total neurons that occurred during $24 \mathrm{~h}$ after transfection of YAP-siRNA or scrambled-siRNA. ${ }^{\star \star} p<0.01(N=3$ wells, $n=10$ visual fields), Wilcoxon's rank sum test. $\mathbf{c}, \mathbf{d}, \mathbf{e}, \mathbf{h}, \mathbf{i}, \mathbf{j}{ }^{\star \star} p<0.01$ ( $N=3$ wells, $n=10$ visual fields) by Wilcoxon's rank-sum test with post-hoc Bonferroni correction. Box plots show the median, quartiles and whiskers that represent $1.5 \times$ the interquartile range. Source data are provided as a "Source Data file".

Live imaging of iPSC-derived neurons. Human normal iPS cells and mutant human iPS cells were plated on a $6 \mathrm{~cm}$ dish with $3 \mu \mathrm{M}$ SB431542, $3 \mu \mathrm{M}$ CHIR99021, and $3 \mu \mathrm{M}$ dorsomorphin, and cultured for 6 days. Next, iPS cells were dissociated into single cells using TrypLE ${ }^{\text {mat }}$ Select (12563-011, Thermo Fisher Scientific, MA, USA) containing $10 \mu \mathrm{M}$ Y27632 (253-00513, Wako, Osaka, Japan). To form neurospheres, the dissociated cells were cultured in KBM medium (16050100, KHOJIN BIO, Saitama, Japan) with $20 \mathrm{ng} / \mathrm{mL}$ Human-FGF-basic (100-18B, Peprotech, London, UK), $10 \mathrm{ng} / \mathrm{mL}$ Recombinant Human LIF (NU0013-1, Nacalai,

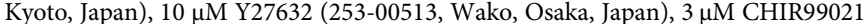
(13122, Cayman Chemical, Ann Arbor, MI, USA), and $2 \mu \mathrm{M}$ SB431542 (13031, Cayman Chemical, Ann Arbor, MI, USA) under suspension culture conditions in a $10 \mathrm{~cm}$ cell-repellent dish. Neurospheres were passaged twice every 7 days, and then dissociated in TrypLE ${ }^{\text {mo }}$ Select containing $10 \mu$ M Y27632 (253-00513, Wako, Osaka, Japan). Dissociated cells were re-seeded onto coverslips coated with poly-Lornithine (P3655, Sigma-Aldrich, St. Louis, MO, USA) and laminin (23016015, Thermo Fisher Scientific, Waltham, MA, USA) in 8-well chambers or 6-well plates with DMEM/F12 (D6421, Sigma-Aldrich, St. Louis, MO, USA) supplemented with B27 (17504044, Thermo Fisher Scientific, Waltham, MA, USA), Glutamax (35050061, Thermo Fisher Scientific, Waltham, MA, USA), and penicillin/streptomycin (15140-122, Thermo Fisher Scientific, Waltham, MA, USA). Two days later, cells were infected with AAV-CMV-YAPdeltaC-ins61 or AAV-CMV-NINS (MOI: 5000) or treated with $20 \mathrm{nM} \mathrm{S1P} \mathrm{(S9666,} \mathrm{Sigma-Aldrich,} \mathrm{MO,} \mathrm{USA).} \mathrm{Six}$ days after viral or drug application, cells were stained with BTA1 (B-9934, SigmaAldrich, St. Louis, MO, USA) and ER-Tracker ${ }^{\mathrm{sm}}$ Red (BODIPY ${ }^{\mathrm{mm}}$ TR Glibenclamide) for $60 \mathrm{~min}$ at $37^{\circ} \mathrm{C}$, and then subjected to live-cell imaging (E34250, Thermo Fisher Scientific, Waltham, MA, USA). Time-lapse images of iPS-derived neurons were acquired at $\times 60$ magnification on an Olympus FV10i-W (Olympus, Tokyo, Japan) at $30 \mathrm{~min}$ intervals for $48 \mathrm{~h}$. The chamber was kept at $37^{\circ} \mathrm{C}$ with $5 \% \mathrm{CO}_{2}$. The ratio of cell death patterns was counted $12 \mathrm{~h}$ after the start of time-lapse image acquisition.

Luciferase assay with iPSC-derived neurons. iPSC-derived neurons $\left(2 \times 10^{4}\right.$ cells) were transfected with $10 \mu \mathrm{g}$ of 8xGTIIC-luciferase reporter (34615, Addgene, Watertown, MA, USA) and $10 \mu \mathrm{g}$ of pGL4.74[hRluc/TK] Vector (E6921, Promega, Madison, WI, USA) using Lipofectamine LTX with Plus Reagent (14338100, Thermo Fisher Scientific, Waltham, MA, USA). The 8xGTIIC-luciferase reporter plasmid possesses eight synthetic TEAD-binding sites upstream of the luciferase gene, making it YAP/TAZ-responsive; this construct was generated by adding four more TEAD-binding sites to 4XGTIIC-Lux, originally created by Ian Farrance ${ }^{58}$ (https://www.addgene.org/34615/). pGL4.74[hRluc/TK] encodes the luciferase reporter gene hRluc (Renilla reniformis). After $48 \mathrm{~h}$ transfection, an equal volume of Dual-Glo Luciferase Reagent (E2920, Promega, Madison, WI, USA) was added to each well. Firefly luminescence was measured on a Spark $10 \mathrm{M}$ multimode microplate reader (TECAN, Männedorf, Switzerland) after incubation for $20 \mathrm{~min}$. For Renilla luminescence, an equal volume of Dual-Glo Stop \& Glo Reagent (E2920, Promega, Madison, WI, USA) was added to each well before Dual-Glo Luciferase Reagent, mixed, and measured on a Spark $10 \mathrm{M}$. For the recovery experiments, AAV-CMV-YAPdeltaC-ins61 or AAV-CMV-NINS (MOI: 5000) or $20 \mathrm{nM}$ S1P (S9666, Sigma-Aldrich, St. Louis, MO, USA) was added to the culture medium 4 days before plasmid transfection.

Electron microscopy of ballooning neurons in mice and human. Mouse and human brain samples were fixed with $2.5 \%$ glutaraldehyde in $0.1 \mathrm{M}$ phosphatebuffered saline (PBS) for $2 \mathrm{~h}$, incubated with $1 \% \mathrm{OsO} 4$ buffered with $0.1 \mathrm{M}$ PBS for $2 \mathrm{~h}$, and dehydrated in a series of graded concentrations of ethanol $(50,70,80,90$, $100,100,100$, and 100\%), and embedded in Epon812 (E14120, science services,
München, Germany). Semi-thin $(1 \mu \mathrm{m})$ sections for light microscopy were collected on glass slides and stained for $30 \mathrm{~s}$ with toluidine blue. Ultrathin $(90 \mathrm{~nm})$ sections were collected on copper grids, double-stained with uranyl acetate and lead citrate. Images were obtained by transmission electron microscopy (H-7100, Hitachi, Hitachinaka, Ibaraki, Japan).

Human samples (cerebral neocortex) was collected at autopsy and directly fixed in $4 \%$ paraformaldehyde two overnight. The sliced brain samples were preserved in $20 \%$ sucrose contained phosphate buffer in $4{ }^{\circ} \mathrm{C}$ until the process for ultrastructural observation. Following procedures were same as mice tissue sample preparation.

Induction of apoptosis and necrosis of primary cortical neurons. Mouse primary cortical neurons were prepared from E17 ICR mouse embryos. Cerebral cortex tissues were rinsed with PBS, and incubated with $0.05 \%$ trypsin in PBS at $37^{\circ} \mathrm{C}$ for $15 \mathrm{~min}$. The cells were passed through a $70 \mu \mathrm{m}$ cell strainer (22-363-548, Thermo Fisher Scientific, MA, USA), collected by centrifugation, and cultured in neurobasal medium (21103049, Thermo Fisher Scientific, Waltham, MA, USA) containing 2\% B27 (17504044, Thermo Fisher Scientific, Waltham, MA, USA), $0.5 \mathrm{mM}$ L-glutamine, and 1\% Penicillin/Streptomycin (15140-122, Thermo Fisher Scientific, Waltham, MA, USA). Forty-eight hours later, the medium was changed to that containing $0.5 \mu \mathrm{M}$ AraC (C3631, Sigma-Aldrich, St. Louis, MO, USA). On Day 4 of primary culture, we added $50 \mathrm{mM}$ glutamate to culture medium for $3 \mathrm{~h}$ to induce apoptosis, or $25 \mu \mathrm{g} / \mathrm{ml} \mathrm{a}$-amanitin (A2263, Sigma-Aldrich, St. Louis, MO, USA) for $48 \mathrm{~h}$ to induce TRIAD necrosis. Neurons were then fixed with $4 \% \mathrm{FA}$ and were subjected to immunohistochemistry. For western blot, we removed culture medium, added $100 \mu \mathrm{L}$ sample buffer $(62.5 \mathrm{mM}$ Tris- $\mathrm{HCl}, \mathrm{pH} 6.8,2 \%$ SDS, $10 \%$ glycerol, $0.0025 \% \mathrm{BPB}, 2.5 \% 2$-mercaptoethanol) to each well of 12 well plate, and recovered the samples. The samples were boiled for $10 \mathrm{~min}$ and subjected to SDSPAGE.

Ischemia induction of cerebral cortex tissues. C57BL/6 J mice were anaesthetized with 1.0\% Isoflurane ${ }^{\circledast}$ (099-06571, FUJIFILM, Osaka, Japan). Body temperature of the mice was maintained at $36.5^{\circ} \mathrm{C} \pm 0.5^{\circ} \mathrm{C}$ during surgery with a heating plate. Skin and hair were disinfected with $70 \%$ ethyl alcohol, and a midline neck incision was made. The common carotid arteries were carefully dissected from fat tissues and the surrounding nerves not to injure vagal nerves, and pulled out with a surgical thread. After obtaining good view of the surgical field, bilateral common carotid arteries were clipped, using a microvascular clip (Dieffenbach Vessel Clip, straight $35 \mathrm{~mm}$, Harvard Apparatus, Holliston, MA, USA) for $10 \mathrm{~min}$ After the surgery, the mice were gently brought back to the cage, and watched carefully until recovered.

Statistics. Box plot is used to depict distribution of observed data, and the data are also plotted as dots. A box plot shows the median, quartiles and whiskers that represent $1.5 \times$ the interquartile range. In the other types of plots, values in each group are summarized by mean \pm S.E.M.

Statistical differences between disease and control groups were evaluated by the Wilcoxon rank-sum test with post-hoc Bonferroni correction. Correlations between HMGB1 concentration and other markers in each individual subject were calculated using Pearson's correlation coefficient.

Ethics. This study was performed in strict accordance with the Guidelines for Proper Conduct of Animal Experiments by the Science Council of Japan. This study was approved by the Committees on Gene Recombination Experiments, Human Ethics, and Animal Experiments of the Tokyo Medical and Dental University (2016-007C6, O2014-005-09, and A2018-153A, respectively). 
Reporting summary. Further information on research design is available in the Nature Research Reporting Summary linked to this article.

\section{Data availability}

The authors declare that the data supporting the findings of this study are available within the article and supplementary information. Full anonymized data will be shared by request from any qualified investigator. The Source Data underlying Figs. 1a, b, 2b, c, e, g, h, 3b, c, 4b, 5b, c, d, e, 6b, c, e, g, i, j, 7b, d, e, f, 8c, 9b, c, d, e, f, g, h, 10c, d, e, h, i, j, k, l, $\mathrm{n}$ and o, and Supplementary Figs. 1, 2, 3, 4a, d, 6a, b, 9b, d, 10b, 11b, d, 12b, 14, 15a, b, $c, d, e$ and $f$ are provided as a Source Data file.

\section{Code availability}

An original program code used to simulate a number of active cell death is available from our Website (http://suppl.atgc.info/041/).

Received: 1 April 2019; Accepted: 19 December 2019; Published online: 24 January 2020

\section{References}

1. Doody, R. S., Farlow, M., Aisen, P. S., Alzheimer's Disease Cooperative Study Data, A. \& Publication, C. Phase 3 trials of solanezumab and bapineuzumab for Alzheimer's disease. N. Engl. J. Med. 370, 1459-1460 (2014).

2. Honig, L. S. et al. Trial of Solanezumab for Mild Dementia Due to Alzheimer's Disease. N. Engl. J. Med. 378, 321-330 (2018).

3. Doody, R. S. et al. A phase 3 trial of semagacestat for treatment of Alzheimer's disease. N. Engl. J. Med. 369, 341-350 (2013).

4. Mullard, A. BACE failures lower AD expectations, again. Nat. Rev. Drug Discov. 17, 385 (2018)

5. Sepulcre, J. et al. Neurogenetic contributions to amyloid beta and tau spreading in the human cortex. Nat. Med. 4, 1910-1918 (2018).

6. Sudol, M. Yes-associated protein (YAP65) is a proline-rich phosphoprotein that binds to the SH3 domain of the Yes proto-oncogene product. Oncogene 9 , 2145-2152 (1994).

7. Strano, S. et al. Physical interaction with Yes-associated protein enhances p73 transcriptional activity. J. Biol. Chem. 276, 15164-15173 (2001).

8. Vassilev, A., Kaneko, K. J., Shu, H., Zhao, Y. \& DePamphilis, M. L. TEAD/TEF transcription factors utilize the activation domain of YAP65, a Src/Yes-associated protein localized in the cytoplasm. Genes Dev. 15, 1229-1241 (2001).

9. Huang, J., Wu, S., Barrera, J., Matthews, K. \& Pan, D. The Hippo signaling pathway coordinately regulates cell proliferation and apoptosis by inactivating Yorkie, the Drosophila Homolog of YAP. Cell 122, 421-434 (2005).

10. $\mathrm{Xu}, \mathrm{M}$. et al. A systematic integrated analysis of brain expression profiles reveals YAP1 and other prioritized hub genes as important upstream regulators in Alzheimer's disease. Alzheimers Dement 14, 215-229 (2018).

11. Zhao, B., Lei, Q. Y. \& Guan, K. L. The Hippo-YAP pathway: new connections between regulation of organ size and cancer. Curr. Opin. Cell Biol. 20, 638-646 (2008).

12. Saucedo, L. J. \& Edgar, B. A. Filling out the Hippo pathway. Nat. Rev. Mol. Cell Biol. 8, 613-621 (2007).

13. Zhao, B., Li, L., Tumaneng, K., Wang, C. Y. \& Guan, K. L. A coordinated phosphorylation by Lats and CK1 regulates YAP stability through SCF(betaTRCP). Genes Dev. 24, 72-85 (2010).

14. Basu, S., Totty, N. F., Irwin, M. S., Sudol, M. \& Downward, J. Akt phosphorylates the Yes-associated protein, YAP, to induce interaction with 14-3-3 and attenuation of p73-mediated apoptosis. Mol. Cell 11, 11-23 (2003).

15. Strano, S. et al. The transcriptional coactivator Yes-associated protein drives p73 gene-target specificity in response to DNA Damage. Mol. Cell 18, 447-459 (2005).

16. Levy, D., Adamovich, Y., Reuven, N. \& Shaul, Y. Yap1 phosphorylation by c$\mathrm{Abl}$ is a critical step in selective activation of proapoptotic genes in response to DNA damage. Mol. Cell 29, 350-361 (2008).

17. Hoshino, M. et al. Transcriptional repression induces a slowly progressive atypical neuronal death associated with changes of YAP isoforms and p73. J. Cell Biol. 172, 589-604 (2006).

18. Mao, Y. et al. The hnRNP-Htt axis regulates necrotic cell death induced by transcriptional repression through impaired RNA splicing. Cell Death Dis. 7, e2207 (2016)

19. Mao, Y. et al. Targeting TEAD/YAP-transcription-dependent necrosis, TRIAD, ameliorates Huntington's disease pathology. Hum. Mol. Genet. 25, 4749-4770 (2016).

20. Tagawa, K. et al. Comprehensive phosphoproteome analysis unravels the core signaling network that initiates the earliest synapse pathology in preclinical Alzheimer's disease brain. Hum. Mol. Genet. 24, 540-558 (2015).
21. Calabrese, B. \& Halpain, S. Essential role for the PKC target MARCKS in maintaining dendritic spine morphology. Neuron 48, 77-90 (2005).

22. Fujita, K. et al. HMGB1, a pathogenic molecule that induces neurite degeneration via TLR4-MARCKS, is a potential therapeutic target for Alzheimer's disease. Sci. Rep. 6, 31895 (2016).

23. Scaffidi, P., Misteli, T. \& Bianchi, M. E. Release of chromatin protein HMGB by necrotic cells triggers inflammation. Nature 418, 191-195 (2002).

24. Lotze, M. T. \& Tracey, K. J. High-mobility group box 1 protein (HMGB1): nuclear weapon in the immune arsenal. Nat. Rev. Immunol. 5, 331-342 (2005).

25. Nakamura, A. et al. High performance plasma amyloid-beta biomarkers for Alzheimer's disease. Nature 554, 249-254 (2018).

26. Arbuzova, A., Schmitz, A. A. \& Vergeres, G. Cross-talk unfolded: MARCKS proteins. Biochem J. 362, 1-12 (2002).

27. Zhang, Y. \& Bhavnani, B. R. Glutamate-induced apoptosis in neuronal cells is mediated via caspase-dependent and independent mechanisms involving calpain and caspase- 3 proteases as well as apoptosis inducing factor (AIF) and this process is inhibited by equine estrogens. BMC Neurosci. 7, 49 (2006)

28. Du, Y. et al. Activation of a caspase 3-related cysteine protease is required for glutamate-mediated apoptosis of cultured cerebellar granule neurons. Proc. Natl Acad. Sci. USA 94, 11657-11662 (1997).

29. Dessi, F., Charriaut-Marlangue, C., Khrestchatisky, M. \& Ben-Ari, Y. Glutamate-induced neuronal death is not a programmed cell death in cerebellar culture. J. Neurochem. 60, 1953-1955 (1993).

30. Silva, M. T. Secondary necrosis: the natural outcome of the complete apoptotic program. FEBS Lett. 584, 4491-4499 (2010).

31. Bergsbaken, T., Fink, S. L. \& Cookson, B. T. Pyroptosis: host cell death and inflammation. Nat. Rev. Microbiol 7, 99-109 (2009).

32. Fink, S. L. \& Cookson, B. T. Caspase-1-dependent pore formation during pyroptosis leads to osmotic lysis of infected host macrophages. Cell Microbiol 8, 1812-1825 (2006).

33. Oakley, H. et al. Intraneuronal beta-amyloid aggregates, neurodegeneration, and neuron loss in transgenic mice with five familial Alzheimer's disease mutations: potential factors in amyloid plaque formation. J. Neurosci. 26, 10129-10140 (2006)

34. Saito, T. et al. Single App knock-in mouse models of Alzheimer's disease. Nat Neurosci. 17, 661-663 (2014).

35. Meng, Z., Moroishi, T. \& Guan, K. L. Mechanisms of Hippo pathway regulation. Genes Dev. 30, 1-17 (2016).

36. Caccamo, A. et al. Necroptosis activation in Alzheimer's disease. Nat. Neurosci. 20, 1236-1246 (2017).

37. Tanaka, $H$. et al. The intellectual disability gene PQBP1 rescues Alzheimer's disease pathology. Mol. Psychiatry 23, 2090-2110 (2018).

38. Kawano, S. et al. A cell-based screening for TAZ activators identifies ethacridine, a widely used antiseptic and abortifacient, as a compound that promotes dephosphorylation of TAZ and inhibits adipogenesis in C3H10T1/2 cells. J. Biochem. 158, 413-423 (2015).

39. Shoji, M. et al. JNK activation is associated with intracellular beta-amyloid accumulation. Brain Res. Mol. Brain Res. 85, 221-233 (2000).

40. LaFerla, F. M., Green, K. N. \& Oddo, S. Intracellular amyloid-beta in Alzheimer's disease. Nat. Rev. Neurosci. 8, 499-509 (2007).

41. Cai, Z. et al. Plasma membrane translocation of trimerized MLKL protein is required for TNF-induced necroptosis. Nat. Cell Biol. 16, 55-65 (2014).

42. Galluzzi, L., Kepp, O. \& Kroemer, G. MLKL regulates necrotic plasma membrane permeabilization. Cell Res. 24, 139-140 (2014).

43. Dondelinger, Y. et al. MLKL compromises plasma membrane integrity by binding to phosphatidylinositol phosphates. Cell Rep. 7, 971-981 (2014).

44. Wang, $H$. et al. Mixed lineage kinase domain-like protein MLKL causes necrotic membrane disruption upon phosphorylation by RIP3. Mol. Cell 54, 133-146 (2014).

45. Yang, Y., Mufson, E. J. \& Herrup, K. Neuronal cell death is preceded by cell cycle events at all stages of Alzheimer's disease. J. Neurosci. 23, 2557-2563 (2003).

46. Mesrouze, Y. et al. Adaptation of the bound intrinsically disordered protein YAP to mutations at the YAP:TEAD interface. Protein Sci. 27, 1810-1820 (2018).

47. Uversky, V. N. Intrinsically disordered proteins and their (disordered) proteomes in neurodegenerative disorders. Front Aging Neurosci. 7, 18 (2015)

48. Hirano, A., Dembitzer, H. M., Kurland, L. T. \& Zimmerman, H. M. The fine structure of some intraganglionic alterations. Neurofibrillary tangles, granulovacuolar bodies and "rod-like" structures as seen in Guam amyotrophic lateral sclerosis and parkinsonism-dementia complex. $J$. Neuropathol. Exp. Neurol. 27, 167-182 (1968).

49. Dickson, D. W. et al. Ballooned neurons in select neurodegenerative diseases contain phosphorylated neurofilament epitopes. Acta Neuropathol. 71, 216-223 (1986)

50. Yamanishi, E. et al. A novel form of necrosis, TRIAD, occurs in human Huntington's disease. Acta Neuropathol. Commun. 5, 19 (2017). 
51. Rebeiz, J. J., Kolodny, E. H. \& Richardson, E. P. Jr. Corticodentatonigral degeneration with neuronal achromasia. Arch. Neurol. 18, 20-33 (1968).

52. Gibb, W. R., Luthert, P. J. \& Marsden, C. D. Corticobasal degeneration. Brain 112, 1171-1192 (1989).

53. Lippa, C. F., Smith, T. W. \& DeGirolami, U. Lobar atrophy with pontine neuronal chromatolysis ("ballooned" neurons). Hum. Pathol. 21, 1076-1079 (1990).

54. Lowe, J. et al. Ballooned neurons in several neurodegenerative diseases and stroke contain alpha B crystallin. Neuropathol. Appl Neurobiol. 18, 341-350 (1992).

55. Mori, H. \& Oda, M. Ballooned neurons in corticobasal degeneration and progressive supranuclear palsy. Neuropathology 17, 248-252 (1997).

56. Sakurai, A. et al. Fragmentation of the Golgi apparatus of the ballooned neurons in patients with corticobasal degeneration and Creutzfeldt-Jakob disease. Acta Neuropathol. 100, 270-274 (2000).

57. Yang, G., Pan, F., Parkhurst, C. N., Grutzendler, J. \& Gan, W. B. Thinnedskull cranial window technique for long-term imaging of the cortex in live mice. Nat. Protoc. 5, 201-208 (2010).

58. Dupont, S. et al. Role of YAP/TAZ in mechanotransduction. Nature 474, 179-183 (2011).

\section{Acknowledgements}

This work was supported by Brain Mapping by Integrated Neurotechnologies for Disease Studies (Brain/MINDS) from the Japan Agency for Medical Research and Development (AMED) (JP18dm0207013h0005); the Strategic Research Program for Brain Sciences (SRPBS) (JP18dm0107057h0002); and a Grant-in-Aid for Scientific Research on Innovative Areas "Foundation of Synapse and Neurocircuit Pathology" (22110001, 22110002) from the Ministry of Education, Culture, Sports, Science and Technology (MEXT) to H.O. This work is also partially supported by Strategic Research Program for Brain Sciences (SRPBS) (JP17dm0107057h0002) to K.T., brain bank supported by AMED (JP18dm0107103) to Y.S., and NIH grant (PO1AG14449) to E.M. We thank Marie Tanaka, Tayoko Tajima, and Emiko Yamanishi (Neuropathology, TMDU), and Naomi Takino and Mika Ito (Jichi Medical University), for technical support. We also thank Prof. Yutaka Hata, (Medical Biochemistry, TMDU) for pLL3.7-ires-GFP-TEADresponsive-H2B-mCherry and RIKEN BioResource Center for providing APP-KI mice.

\section{Author contributions}

H.T., K.K., K.F.: acquisition of data, drafting of manuscript, H.H.: analysis of data, drafting of manuscript. S.Y., X.J.: acquisition of data. M.W.: collection of samples, acquisition and analysis of data, drafting of manuscript. K.T.: acquisition of data. G.O.: collection of samples. A.I.: acquisition and analysis of data. N.A.: collection of samples.
M.K., N.T., K.F., E.M.: collection of samples. H.A.: collection of samples, acquisition and analysis of data. T.S. and T.S.: collaboration on the mouse model. M.S.: discussion and suggestion for manuscript. Si.M.: collaboration on AAV. S.S., H.Okano., G.S., Y.S., S.M.: collection of samples, acquisition and analysis of data. H.Okazawa.: conception and design of the study, arrangement of collaboration, obtaining funding, drafting of manuscript.

\section{Competing interests}

Shingo Yamada is an employee of Shino-Test Corporation. Hitoshi Okazawa and all other authors have nothing to report.

\section{Additional information}

Supplementary information is available for this paper at https://doi.org/10.1038/s41467020-14353-6.

Correspondence and requests for materials should be addressed to H.O.

Peer review information Nature Communications thanks Yong-Gang Yao and the other, anonymous, reviewer(s) for their contribution to the peer review of this work. Peer reviewer reports are available.

Reprints and permission information is available at http://www.nature.com/reprints

Publisher's note Springer Nature remains neutral with regard to jurisdictional claims in published maps and institutional affiliations.

(c) (i) Open Access This article is licensed under a Creative Commons Attribution 4.0 International License, which permits use, sharing, adaptation, distribution and reproduction in any medium or format, as long as you give appropriate credit to the original author(s) and the source, provide a link to the Creative Commons license, and indicate if changes were made. The images or other third party material in this article are included in the article's Creative Commons license, unless indicated otherwise in a credit line to the material. If material is not included in the article's Creative Commons license and your intended use is not permitted by statutory regulation or exceeds the permitted use, you will need to obtain permission directly from the copyright holder. To view a copy of this license, visit http://creativecommons.org/ licenses/by/4.0/.

(c) The Author(s) 2020 